\title{
Modification de couleur du bois de chênes européens exposés à la lumière solaire
}

\author{
JF Mazet 1, MC Triboulot-Trouy 2, A Merlin 2,*, \\ G Janin 3, X Deglise 2
}

\author{
1 Service des brevets de la Communauté européenne, 2280 HV Rijwijk, Pays-Bas; \\ 2 Laboratoire de photochimie appliquée, ESSTIB, Université de Nancy I, \\ BP 239, 54506 Vandceuvre-lès-Nancy Cedex; \\ ${ }^{3}$ Station de recherches sur la qualité des bois, CRF INRA, Champenoux, \\ 54280 Seichamps, France
}

(Reçu le 12 mai 1992; accepté le 15 octobre 1992)

\begin{abstract}
Résumé - L'étude du comportement photochimique du bois d'Abies grandis caractérisé par la quasi-absence de substances extractibles colorées, a permis d'établir un modèle mécanistique capable de rendre compte à l'échelle moléculaire des modifications de couleur du bois exposé à une lumière de type solaire. Ce modèle a été étendu au bois de chênes européens (Quercus robur et petraea), essences riches en substances extractibles colorées et pour lesquelles les caractéristiques de couleur sont un critère de qualité. En plus du jaunissement observé et analysé sur le bois d'Abies grandis, un éclaircissement correspondant à une diminution de l'absorption sur le domaine spectral 500 à $600 \mathrm{~nm}$ a été noté. Cette décoloration a pu être imputée, au moins en partie, à la photodégradation des lignines.
\end{abstract}

\section{Quercus robur / Quercus petraea / lignine / photodégradation / couleur / spectroscopie}

Summary - Wood photodiscoloration of European oak under solar light exposure. A previous study concerning the photochemistry of Abies grandis wood, a species which is characterized by the very low content of colored extractable substances, has made it possible to establish a mechanical model (fig 22) to take into account the discoloration of wood under solar-type light exposure at the molecular level. In this paper, this model was extended to European species of oak (Quercus robur and petraea) with high levels of colored extractable substances and for which the color characteristics are a quality criterion.

In addition to the observed and analyzed yellowing in Abies grandis wood, a color lightening corresponding to a lowered absorption over the spectral range $(500-600 \mathrm{~nm}$ ) (figs 2, 6) has been observed. Lignin photodegradation is certainly in part responsible for this decoloration.

It has also been shown that oak sapwood and heartwood undergo different kinds of photodecoloration (fig 3). For color photostabilization of oak wood under solar light, the results indicate that it is necessary to use UV absorbers to lower the yellowing due to UV light and antioxidants to inhibit color lightening due to reactions with oxygen.

Quercus robur / Quercus petraea / lignin / photodecoloration / FTIR absorption spectroscopy / $U V$ and visible spectroscopy

\footnotetext{
* Correspondance et tirés à part
} 


\section{INTRODUCTION}

La couleur d'un bois est susceptible de varier sous l'action de facteurs externes liés à l'environnement (lumière, humidité, température...). Lors de l'utilisation en décoration intérieure (ameublement, panneaux de revêtements muraux...), les modifications de la couleur naturelle des placages ont essentiellement pour origine la lumière solaire. Cette altération de l'aspect du bois est imputable à une réaction de surface : la pénétration du rayonnement ultraviolet ne dépasse pas $75 \mu \mathrm{m}$ et la lumière visible peut aller jusqu'à $200 \mu \mathrm{m}$ (Browne et Simonson, 1957; Hon et Ifju, 1978). En revanche, les modifications de couleur peuvent affecter le matériau plus en profondeur à la suite de réactions secondaires de transfert d'énergie (LablacheCombier, 1985).

Cette photodégradation est observée aussi bien pour des bois recouverts d'une finition transparente (Gaillard, 1984) que pour ceux qui sont exposés à l'état brut (Dirckx, 1988; Mazet, 1985).

L'étude des modifications moléculaires qui s'of èrent lors d'une irradiation de type solaire au sein de la structure du bois de sapin de Vancouver (Abies grandis L) nous a montré les rôles essentiels joués par le rayonnement ultraviolet, la lignine et l'oxygène de l'air dans le jaunissement intense observé sur cette essence (Dirckx, 1988, op cit, Dirckx et al, 1922).

Nous avons poursuivi ce travail en nous intéressant au comportement photochimique du bois de chêne, essence de tranchange dont les caractéristiques de couleur sont un critère de qualité. Le but cette étude fondamentale est d'établir les mécanismes de la photodégradation de ce matériau afin d'envisager des remèdes adaptés à sa protection à la lumière; en effet, l'amélioration de la durabilité de l'aspect naturel des placages de bois de chêne présente un réel impact économique.

Notre démarche a consisté à étudier globalement la photodégradation du bois de chêne en cherchant à la comparer aux résultats obtenus sur le bois de sapin de Vancouver, essence qui constitue un modèle simple pour l'étude du comportement photochimique du bois et pour laquelle nous avons pu proposer un schéma des mécanismes moléculaires de sa photodégradation (Dirckx, 1988, Dirckx et al, 1990; Dirckx et al, 1992).

\section{MATÉRIEL ET MÉTHODES}

\section{Espèces utilisées}

Nous avons travaillé sur des échantillons de bois de chênes européens (Quercus robur $L$ et Quercus petraea (Matt) Liebl, sans distinction), provenant de différentes grumes des parcs d'une part des établissements Ober-France, et d'autre part de la régie de l'Office national des forêts de Sarre-Union.

Par ailleurs, nous avons mené en parallèle des essais avec des échantillons de bois d'Abies grandis $L$ pour pouvoir comparer le comportement du bois de chêne exposé à la lumière solaire à celui de l'essence modèle du comportement photochimique du bois.

\section{Préparation des échantillons}

Deux types d'échantillons ont été réalisés en fonction de la technique spectroscopique qui doit leur être appliquée pour suivre la photodégradation :

- échantillons sous forme de coupes minces de $20 \times 10 \mathrm{~mm}$ et dont l'épaisseur peut varier de 50 à $80 \mu \mathrm{m}$ : ces coupes de bois de chêne ont été préparées sur un microtome Reichert type OME dans le plan LT (longitudinal - tangentiel) et dans le bois final, afin d'éviter autant que possible des fissures dues à la présence de gros vaisseaux; du fait de la structure du bois d'Abies 
grandis, les coupes pour cette essence ont été réalisées dans le bois initial car celui-ci est beaucoup plus tendre;

- échantillons massifs d'environ $20 \times 10 \times 3 \mathrm{~mm}$ selon les directions LTR : la face LT a été aplanie préalablement au microtome; des échantillons de placage industriel de bois de chêne (épaisseur $0,6 \mathrm{~mm}$ ) ont également été utilisés lors de certains essais.

\section{Dispositif d'irradiation}

Les modifcations de la couleur d'un bois mis en cuvre en décoration intérieure ont le plus souvent pour origine la lumière du jour. Les radiations solaires atteignant la surface de la terre s'étendent de l'ultraviolet $(290 \mathrm{~nm})$ à l'infrarouge (2 $300 \mathrm{~nm}$ ) (Bird et al, 1982). La région ultraviolette $(290$ à $400 \mathrm{~nm})$ ne représente en fait que $6 \%$ de l'énergie totale du rayonnement solaire. La répartition spectrale dans le domaine ultraviolet et visible est soumise à de nombreux paramètres, comme la région du globe terrestre considérée, les saisons, les variations climatiques... Aussi, pour standardiser les irradiations et accélérer la photodégradation, nous avons simulé la lumière du jour à l'aide d'une lampe à vapeur de mercure haute pression du type Hanau TQ 150. Ce brûleur est équipé d'une jaquette en pyrex qui élimine les radiations de longueur d'onde supérieure à $310 \mathrm{~nm}$.

Le flux lumineux moyen émis par cette source à la distance utilisée pour l'exposition des échantillons mesuré à $360 \mathrm{~nm}$ à l'aide d'un radiomètre est de $5 \mathrm{~mW} / \mathrm{cm}^{2}$. Notons qu'à cette longueur d'onde, le flux émis par le soleil à la surface de la terre est d'environ $0,1 \mathrm{~mW} / \mathrm{cm}^{2}$ (Lablache-Combier, 1985).

Pour séparer les effets du rayonnement ultraviolet et ceux de la lumière visible, nous avons utilisé un filtre passe-haut coupant à $400 \mathrm{~nm}$.

\section{Analyse spectroscopique}

Suivant la nature des échantillons, nous avons mis en œuvre deux techniques d'analyse spectroscopique : absorption pour les coupes et réflexion pour les échantillons massifs.

\section{Spectroscopie d'absorption infrarouge}

Nous avons suivi l'absorption des coupes de bois grâce à un spectromètre à transformée de Fourier (FTIR 1750, Perkin-Elmer) qui permet des analyses quantitatives correctes jusqu'à une densité optique de 3 .

\section{Spectroscopie d'absorption et de réflexion ultraviolet-visible}

Pour ces deux modes d'utilisation, nous avons adjoint à un spectromètre Perkin-Elmer Lambda 3 une sphère d'intégration enduite de sulfate de baryum permettant de collecter la lumière dispersée dans toutes les directions, et ainsi de travailler avec des échantillons diffusant la lumière. Avec les coupes minces, l'échantillon est placé à l'entrée de la sphère (fig 1A); nous obtenons alors un spectre d'absorption diffuse. Les échantillons massifs sont en revanche placés à l'arrière du dispositif (fig 1B). Deux traitements sont alors possibles: analyse de la réflexion diffuse et mesure de couleur avec le calcul des valeurs tristimulaires $X Y$ et $Z$ sous l'illuminant D65 avec un angle d'ouverture de $2^{\circ}$.

Cette sphère d'intégration permet l'analyse du domaine spectral de 250 à $700 \mathrm{~nm}$.

Ces spectromètres ultraviolet-visible et FTIR sont couplés à des stations de données qui faci-
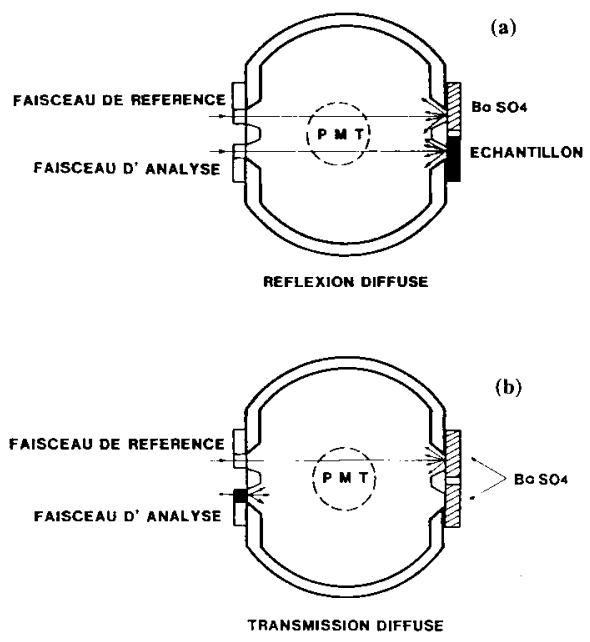

Fig 1. Schéma de principe du fonctionnement de la sphère d'intégration. 
litent l'analyse des spectres en particulier en permettant l'obtention des courbes de différence de 2 spectres. Elles ont aussi rendu possible la normation de spectres d'absorption infrarouge ont ramenés à une valeur identique de la densité optique à $1800 \mathrm{~cm}^{-1}$, longueur d'onde où aucun des constituants du bois n'absorbe. Aucune normation n'a été nécessaire pour les spectres d'absorption et de réflexion ultravioletvisible.

Pour apprécier le rôle de l'oxygène dans les phenomènes de photocoloration du bois, nous avons conçu des cellules permettant l'irradiation et l'enregistrement des spectres d'absorption infrarouge et ultraviolet dans différentes atmosphères gazeuses. Ces dispositifs ont été décrits dans un travail précédent (Dirckx et al, 1992).

Pour tenir compte de la variabilité de la couleur des échantillons liée à leur nature et à leur état de surface, nous avons pris en compte les modifications de l'absorption UV-visible et infrarouge au cours d'une même irradiation de 5 échantillons différents.

\section{RÉSULTATS}

Les modifications de couleur qui interviennent lors de l'exposition du bois de chêne au rayonnement solaire peuvent être quantifiées dans le système CIE. Lab (Janin, 1986; Decarreau, 1988; Flot, 1988; Dordet, 1990) en suivant, en fonction du temps d'irradiation, les variations de la luminance $L$ et des coordonnées $a$ et $b$ qui correspondent respectivement aux 2 couples de couleurs complémentaires rouge-vert et bleu-jaune.

Des échantillons massifs de bois de chêne (aubier et duramen) ont été exposés plus de $300 \mathrm{~h}$ sous un flux moyen de 5 $\mathrm{mW} / \mathrm{cm}^{2}$ à $360 \mathrm{~nm}$. La variation de luminance (fig 2a) permet de distinguer 2 phénomènes. Durant les 10 premières heures d'exposition, il se produit une baisse importante et rapide de la luminance pour l'aubier et le duramen. Les évolutions de ces bois diffèrent ensuite : tandis que l'aubier s'éclaircit très rapidement jusqu'à dé- passer au bout d'une centaine d'heures d'irradiation la valeur initiale de la luminance, le duramen continue à s'assombrir et ce n'est qu'après $200 \mathrm{~h}$ d'exposition que le processus s'inverse lentement. Les changements de tonalité exprimés dans le plan (a,b) (fig 2b) montrent que ces échantillons jaunissent lors de l'irradiation, $b$ augmentant fortement. Les variations sont plus accentuées sur l'aubier que sur le duramen.

Dans l'espace CIE Lab de représentation des couleurs, la différence totale de couleur $\Delta \mathrm{E}$ entre 2 échantillons est donnée par la relation:

$$
\Delta E=\sqrt{\Delta \mathrm{L}^{2}+\Delta \mathrm{a}^{2}+\Delta \mathrm{b}^{2}}
$$

où $\Delta \mathrm{L}, \Delta \mathrm{a}$ et $\Delta \mathrm{b}$ sont les différences respectives en luminance et coordonnées chromatiques entre les 2 échantillons. Sur la figure 3 , nous avons porté l'évolution avec le temps d'irradiation de la différence totale de couleur $\Delta \mathrm{E}$ entre l'aubier le duramen de bois de chêne. Cette différence, déjà élevée au départ $(\Delta E=6,5)$ ne fait que s'accentuer pour atteindre un palier $(\Delta \mathrm{E}=14$ après $2000 \mathrm{~h}$ d'irradiation). Ce résultat montre la nécessité d'éliminer l'aubier de la fabrication des panneaux décoratifs, des meubles, des parquets... En effet, au lieu d'atténuer le contraste, la lumière du jour ne fera que la renforcer.

Nous avons également soumis à une exposition prolongée dans les mêmes conditions 7 échantillons de placage de bois de chêne provenant d'arbres distincts et présentant des caractéristiques de couleur bien distinctes, aussi bien en ce qui concerne la luminance $L$ que les coordonnées chromatiques a et b. Après $940 \mathrm{~h}$ (fig 4), nous constatons que les 7 échantillons ont conservé leur différence de couleur. Là encore, l'effet de l'irradiation continue n'a pas été de gommer les écarts existant au départ. Ce résultat justifie le fait d'utiliser des placages présentant des 

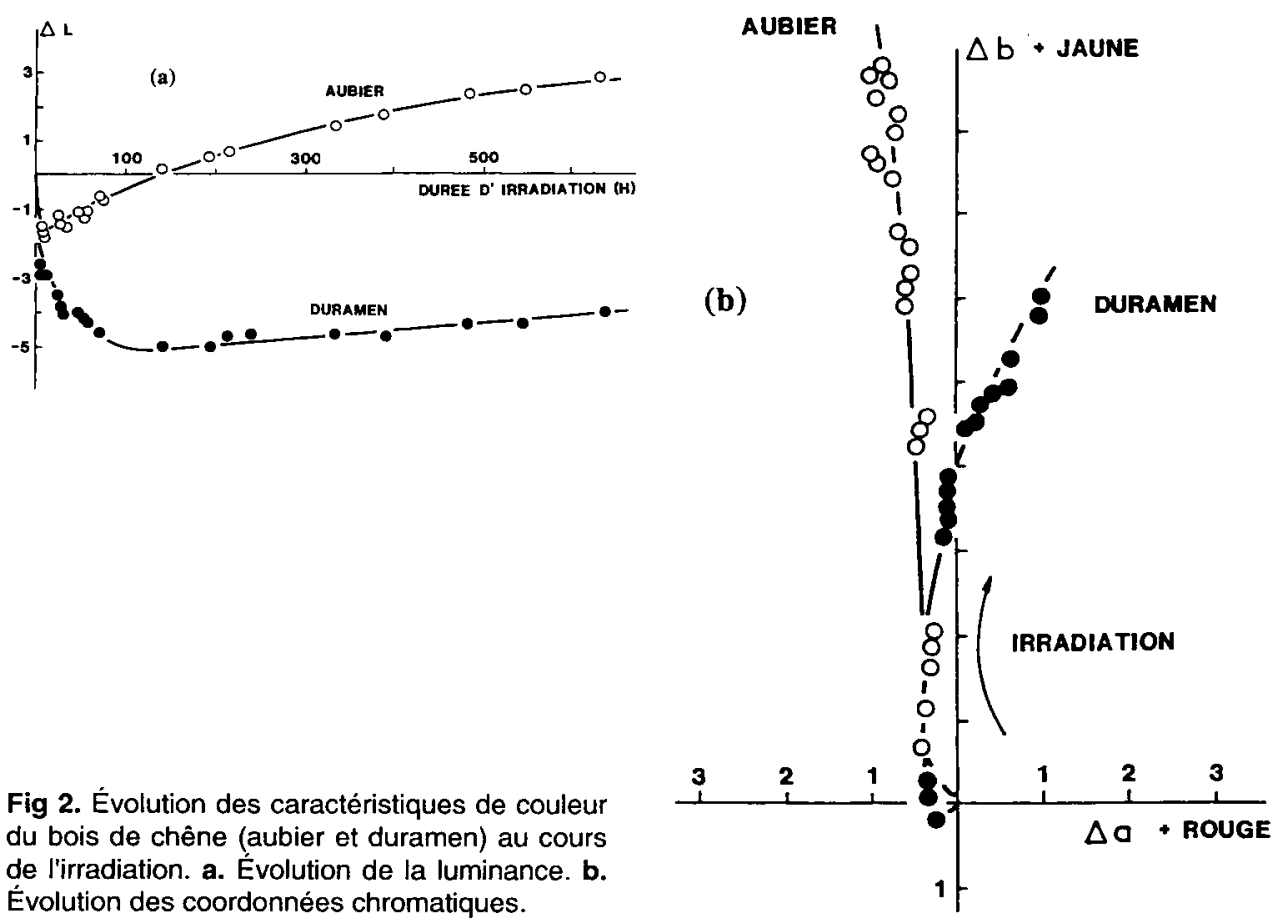

Fig 2. Évolution des caractéristiques de couleur du bois de chêne (aubier et duramen) au cours de l'irradiation. a. Évolution de la luminance. $b$. Évolution des coordonnées chromatiques.

caractéristiques de couleur voisines pour réaliser un ensemble décoratif d'intérieur. Ainsi, même si la couleur se modifie au cours du temps, I'uniformité devrait être conservée.

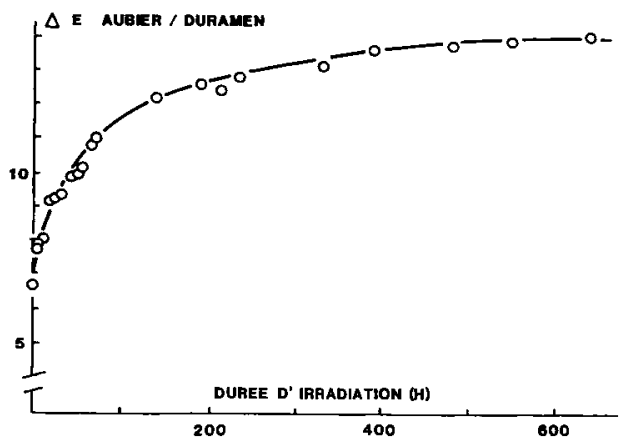

Fig 3. Évolution de la différence total en couleur entre aubier et duramen du bois de chêne en fonction du temps d'irradiation.

Les mesures dans le système CIE Lab représentant la modification globale de l'aspect coloré des échantillons par intégration sur tout le domaine de sensibilité de l'œil ne permettent pas une interprétation des phénomènes à l'échelle moléculaire. Une analyse spectrale des évolutions de l'absorption avec le temps d'irradiation dans les domaines ultraviolet et infrarouge est nécessaire.

\section{Suivi de la photodégradation du bois de chêne par spectroscopie d'absorption ultraviolet-visible}

La spectroscopie d'absorption ultravioletvisible a été souvent utilisée pour suivre la photodégradation du bois (Kalnins, 1966; 


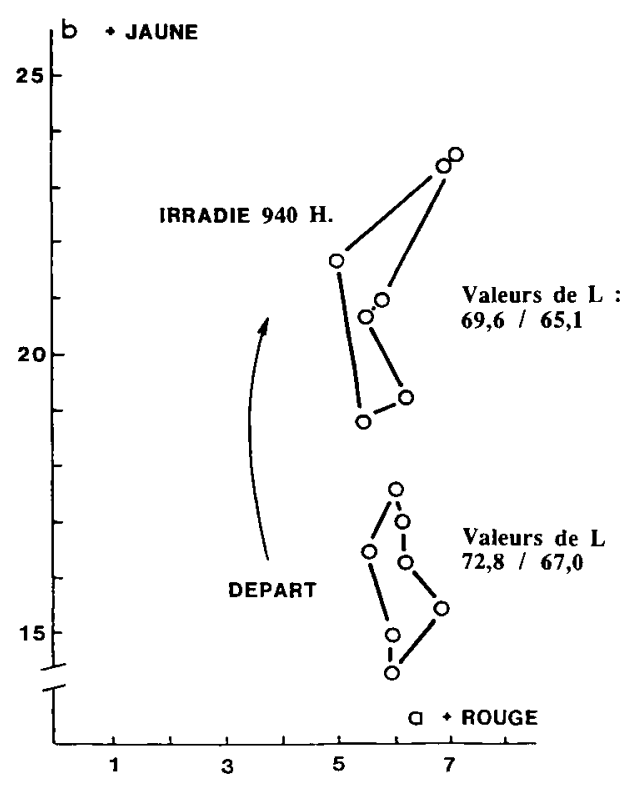

Fig 4. Évolution, en fonction du temps d'irradiation, des caractéristiques $a$ et $b$ et $L$ de 7 placages de bois de chêne différents au départ.

Musha et Goring, 1975; Dirckx et al, 1992). Tous ces auteurs ont noté l'aspect similaire du spectre du bois avec celui de la lignine isolée, ce qui conduit à conclure que l'absorption de la lumière ultraviolette par le bois est principalement due à l'absorption des lignines.

Les échantillons utilisés pour cette étude sont des coupes minces, dont l'épaisseur varie de 40 à $60 \mu \mathrm{m}$. Les spectres d'absorption ultraviolet-visible d'une coupe de duramen de bois de chêne non irradié (fig 5a) et irradiée 1400 h (fig $5 b)$ présentent une structure continue et peu résolue, rendant leur exploitation difficile. Pour mieux révéler les zones spectrales qui évoluent durant l'irradiation, nous pouvons construire les courbes de différence obtenues par la soustraction du

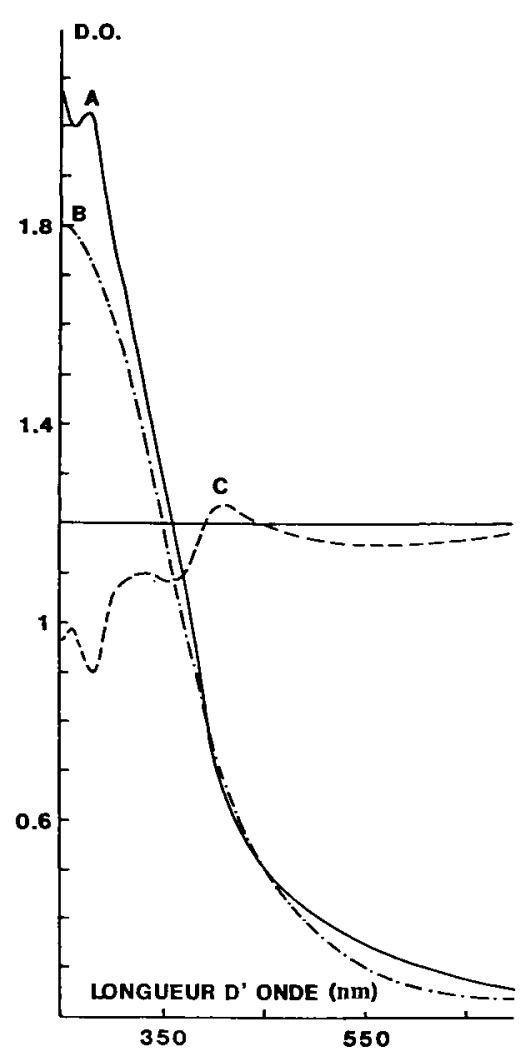

Fig 5. Spectres d'absorption ultraviolet-visible d'une lamelle de bois de chêne. A. Spectre de départ. B. Spectre après irradiation. C. Courbe de différence des 2 spectres.

spectre après irradiation au spectre de la coupe non irradiée. Cette courbe de différence (fig $5 \mathrm{c}$ ) fait ressortir dans le domaine visible une large bande de décoloration centrée à $580 \mathrm{~nm}$ (variation négative) et une bande de coloration vers $415 \mathrm{~nm}$ (variation positive); et dans le domaine ultraviolet, plusieurs bandes : à $360 \mathrm{~nm}$ (variation négative), à $330 \mathrm{~nm}$ (variation positive), à $280 \mathrm{~nm}$ (variation négative) à $260 \mathrm{~nm}$ (variation positive). 
Cette longue irradiation donne la tendance finale de la modification de la couleur du duramen de bois de chêne.

Pour décrire la chronologie des différentes étapes qui interviennent dans la modification de l'absorption ultravioletvisible des échantillons, nous avons suivi l'évolution de ces courbes de différence avec le temps d'irradiation (fig 6).

Dès le début de l'irradiation (fig 6a: $4 \mathrm{~h}$ d'exposition), on note une augmentation générale de l'absorption dans le domaine visible avec un maximum vers $470 \mathrm{~nm}$, tandis que dans l'ultraviolet, la baisse de l'absorption à $350 \mathrm{~nm}$ indique la disparition de chromophores absorbant à cette longueur d'onde.

Après $47 \mathrm{~h}$ (fig 6b), l'absorption dans le visible ne cesse d'augmenter, avec un maximum vers $410 \mathrm{~nm}$. La diminution de la bande à $350 \mathrm{~nm}$ se poursuit et des chromophores absorbant vers $305 \mathrm{~nm}$ sont créés. Sur les figures $7 a$ et $b$, nous avons tracé respectivement l'évolution des absorptions à $470 \mathrm{~nm}$ et à $350 \mathrm{~nm}$ d'une coupe de duramen de bois de chêne en début d'irradiation. La forte pente à l'origine de la figure $7 \mathrm{~b}$ montre que la première phase semble être la photodégradation des produits absorbant à $350 \mathrm{~nm}$. L'apparition progressive et continue de chromophores absorbant dans le visible $(470 \mathrm{~nm})$ constitue la deuxième étape du processus. Le maximum à $410 \mathrm{~nm}$ apparaît pour des durées d'irradiation plus longues (fig 8). Après $21 \mathrm{~h}$ d'exposition, les 2 maxima sont bien differentiés et celui à $410 \mathrm{~nm}$ devient alors prépondérant.

Après $268 \mathrm{~h}$ (fig 6c), une bande positive apparaît à $260 \mathrm{~nm}$. Dans la région visible, outre l'apparition de produits colorés absorbant vers $410 \mathrm{~nm}$, il se produit une diminution générale de la différence de densité optique sur l'ensemble de la zone

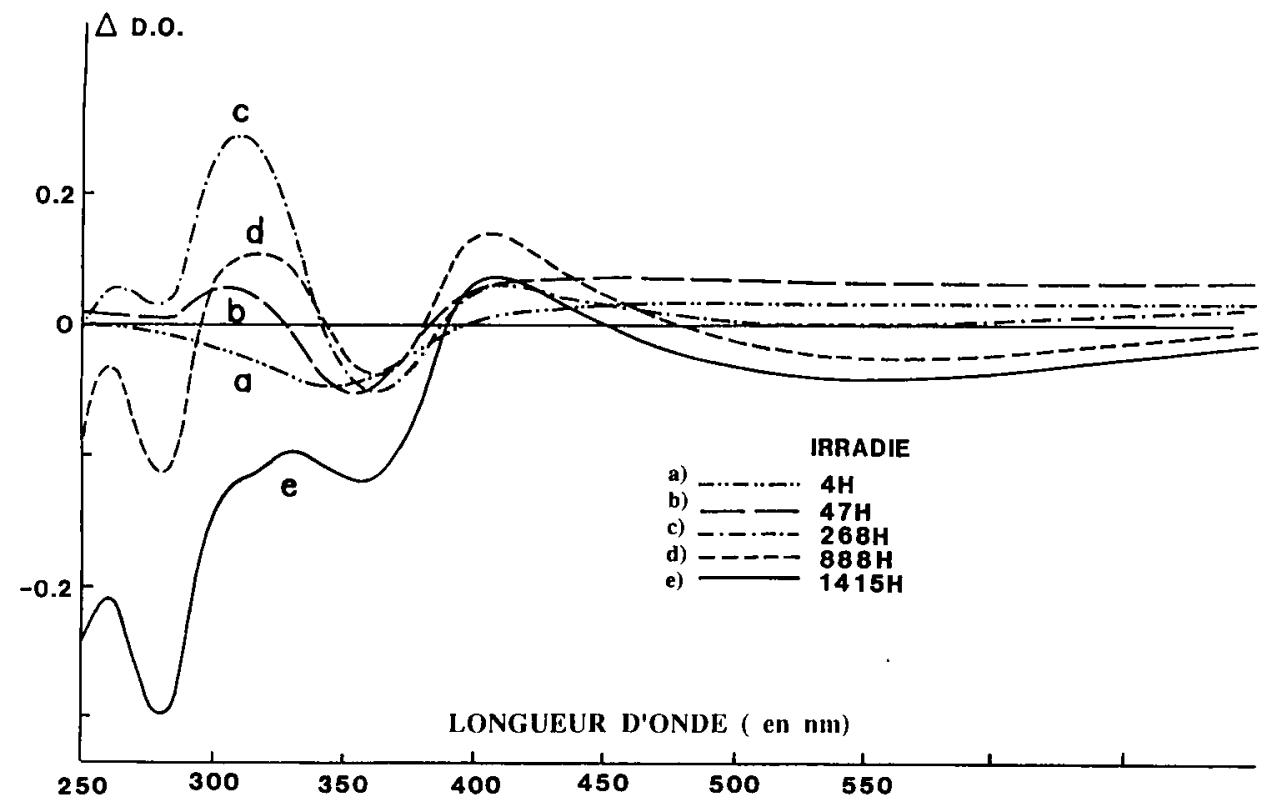

Fig 6. Évolution de la courbe de différence des spectres après/avant irradiation en fonction du temps. 

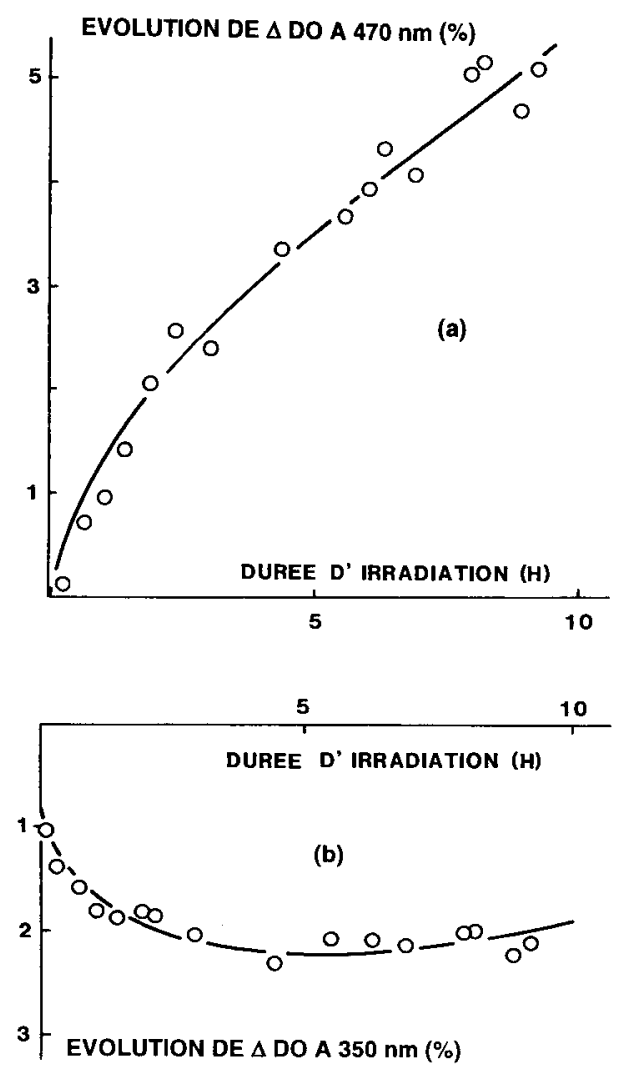

Fig 7. Duramen de bois de chêne : évolution des bandes d'absorption situées vers $470 \mathrm{~nm}$ (a) et $350 \mathrm{~nm}$ (b) en fonction du temps d'irradiation.

allant de $450 \mathrm{~nm}$ à $700 \mathrm{~nm}$, qui révèle la dégradation d'autres produits colorés. Les chromophores absorbant vers $305 \mathrm{~nm}$ continuent d'être générés. Par contre, la diminution de l'absorption à $350 \mathrm{~nm}$ semble ralentie; on peut penser qu'elle est contrariée par la croissance importante des 2 bandes qui l'entourent.

Après 888 h (fig 6d), les évolutions de l'absorption dans le domaine visible (bande de décoloration de 500 à $700 \mathrm{~nm}$ et coloration à $410 \mathrm{~nm}$ ) se sont encore ac- centuées. La diminution de l'absorption à $280 \mathrm{~nm}$ est très intense et entraîne la chute des bandes positives voisines.

L'allure de cette courbe de différence est analogue à celle que l'on avait obtenue après 1400 heures d'irradiation (fig 5c)/ Ces résultats peuvent être reliés aux modifications de couleur que nous avons quantifiées dans le système CIE Lab :

- l'augmentation initiale de l'absorption correspond à la chute brutale de la luminance en début d'irradiation (assombrissement de l'échantillon);

- la baisse générale de l'absorption dans la zone 500 à $700 \mathrm{~nm}$ est directement liée à l'augmentation progressive de la luminance au bout d'une centaine d'heures d'irradiation (éclaircissement de l'échantillon);

- la création de produits absorbant à 470 $\mathrm{nm}$ puis vers $410 \mathrm{~nm}$ est en relation avec le jaunissement des coupes (augmentation de b).

Des observations similaires ont été notées lors de l'irradiation à la lumière solaire d'échantillons de bois d'Abies grandis (Dirckx, 1988). En début d'irradiation, il a été constaté une croissance de l'absorption à $430 \mathrm{~nm}$; ensuite, ce maximum d'absorption croît en se déplaçant jusqu'à 410 $\mathrm{nm}$. Dans le cas du bois de chêne, le premier maximum se situant vers $470 \mathrm{~nm}$ et le deuxième à $410 \mathrm{~nm}$, il ne peut s'agir d'un effet bathochrome (déplacement de la bande d'absorption vers les courtes longueurs d'onde) mais bien de 2 bandes distinctes. Ce sont probablement 2 produits différents également dans le cas du bois de sapin de Vancouver, mais du fait de la proximité de leurs maxima, la résultante se présente sous forme d'une bande unique. L'absorption à $430 \mathrm{~nm}$ a été attribuée à des radicaux libres du type phenoxy dans le cas du bois d'Abies grandis (Dirckx et al, 1992). Lors de l'irradiation du bois de chêne, on peut penser que les radicaux libres générés ont une structure chimique 


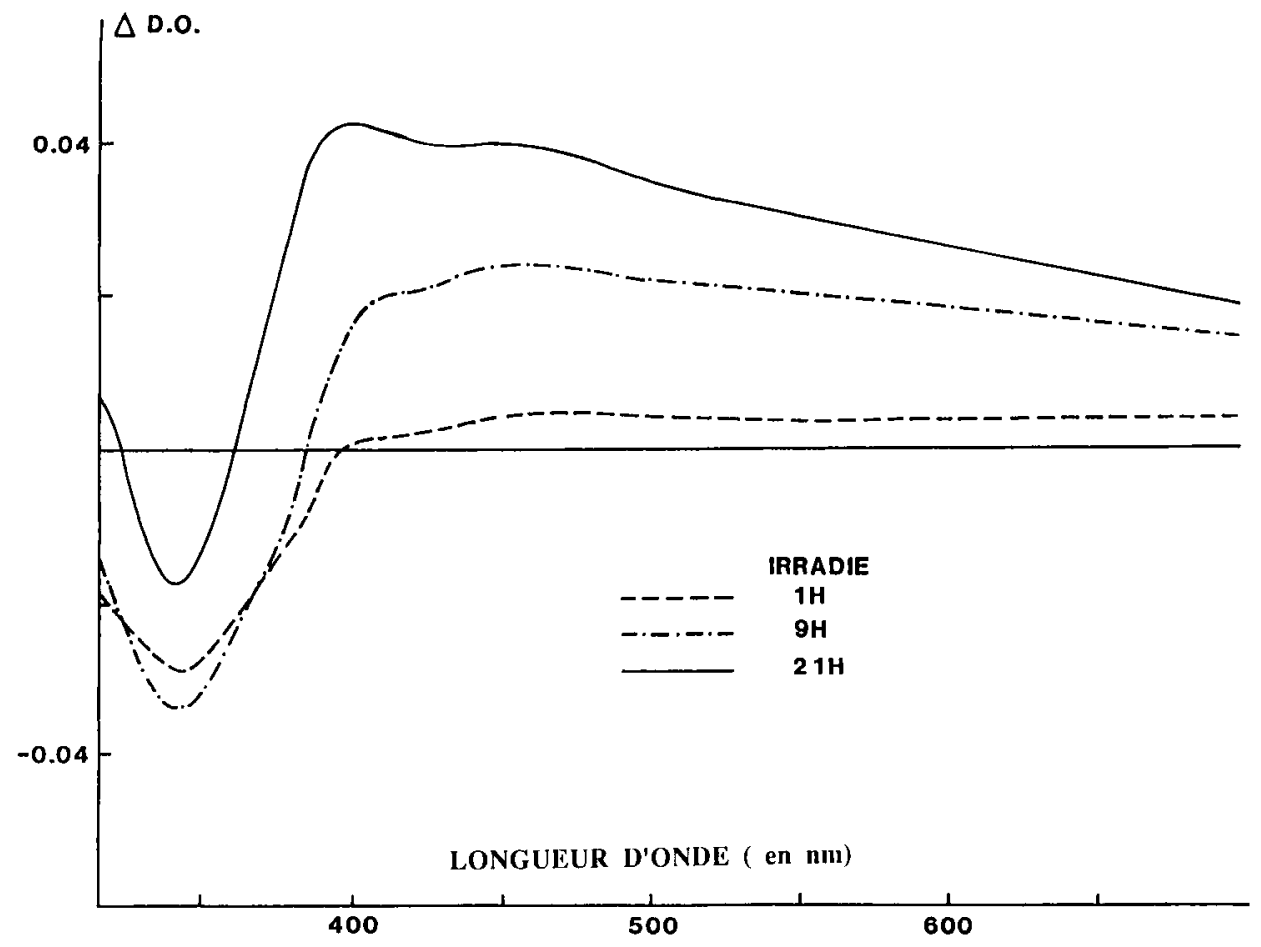

Fig 8. Courbe de différence des spectres après/avant irradiation pour du duramen de bois de chêne : mise en évidence des 2 maximums d'absorption (470 nm et $410 \mathrm{~nm}$ ).

différente, avec une délocalisation des électrons plus importante responsable de l'absorption à $470 \mathrm{mn}$.

En revanche, aucune bande de décoloration dans le visible n'a été observée lors de l'irradiation du bois d'Abies grandis. Ce photoéclaircissement a été constaté lors de l'irradiation du bois de hêtre (Schmitt, 1984) et de duramen des bois de cèdre rouge (Juniperus virginiana $\mathrm{L}$ ) et de noyer noir américain (Juglans nigra L) (Mc Ginnes, 1975).

La nette décroissance de l'absorption à $280 \mathrm{~nm}$ traduit une dégradation de la lignine. Cette diminution continue de la den- sité optique à cette longueur d'onde a été suivie en microscopie ultraviolette de coupes minces de bois (Scott et Goring, 1970). Après 2 h seulement, ces auteurs observent une chute de l'absorption à $280 \mathrm{~nm}$ de $25 \%$ par rapport à sa valeur initiale.

\section{Suivi de la photodégradation du bois de chêne par spectroscopie d'absorption infrarouge}

Sur la figure 9, nous avons comparé les spectres d'absorption infrarouge des 


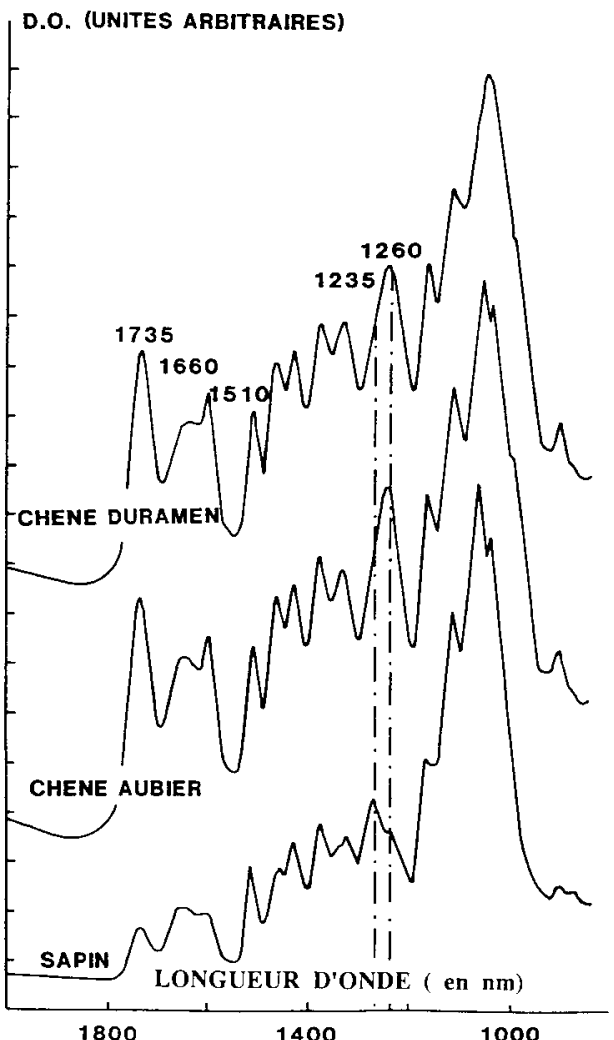

Fig 9. Spectres infrarouges de bois de chêne (aubier et duramen) et de bois de sapin de Vancouver.

coupes de duramen et d'aubier de bois de chêne et d'Abies grandis. Si l'aspect général des 3 spectres est comparable, les intensités relatives de certaines bandes d'absorption présentent des différences notables d'une essence à l'autre. Dans le cas du bois de sapin de Vancouver qui contient essentiellement de la lignine riche en unités gaïacyles, la bande à $1265 \mathrm{~cm}^{-1}$ est nettement plus intense que celle à $1235 \mathrm{~cm}^{-1}$. En revanche pour le bois de chêne dont la lignine contient autant de motifs syringles que gaïacyles, l'intensité de la bande à $1235 \mathrm{~cm}^{-1}$ est la plus forte.

En confrontant le spectre d'absorption infrarouge $\mathrm{du}$ bois de chêne à ceux d'autres essences et des constituants principaux du bois (Marchessault, 1962; Bolker et Sommerville, 1963; Michell et al, 1969; Chow, 1971; Nagaty et al, 1982; Pecina, 1982; Dirckx, 1988), nous avons pu attribuer la totalité des bandes d'absorption et déterminer le ou les constituants concernés (tableau I).

Les modifications qui interviennent dans le spectre d'absorption infrarouge d'une coupe de duramen de bois de chêne lors d'une irradiation de type solaire (fig 10) sont principalement localisées dans la région spectrale $1800-1500 \mathrm{~cm}^{-1}$ :

- forte augmentation du pic à $1735 \mathrm{~cm}^{-1}$ (fig 11a);

- augmentation du massif situé vers 1640 $\mathrm{cm}^{-1}$;

- diminution des pics à 1600 et $1504 \mathrm{~cm}^{-1}$ (fig 11b).

On a également constaté la disparition de la bande à $1460 \mathrm{~cm}^{-1}$ et une baisse générale de l'absorption sur tout le reste du spectre.

La croissance de l'absorption à 1735 $\mathrm{cm}^{-1}$ et parallèlement la diminution à 1265 et $1504 \mathrm{~cm}^{-1}$ ont également été observées sur du pin (Pinus spp) exposé aux rayonnements ultraviolets supérieurs à 220 nm (Hon, 1983; Hon et Chang, 1984). Ces auteurs interprètent ces variations comme résultant respectivement d'une oxydation de la cellulose et de la lignine (augmentation des groupements carbonyls) et d'une altération des structures aromatiques de la lignine par le rayonnement ultraviolet.

L'évolution des spectres d'absorption infrarouge du bois d'Abies grandis au cours de l'irradiation (Dirckx, 1988; Dirckx et al, 1992) est comparable à celle observée 
Tableau I. Comparaison des spectres d'absorption infrarouge des bois de chêne et de sapin de Vancouver : localisation et attribution des bandes d'absorption infrarouge des 2 essences (Marchessault, 1962; Pecina, 1982).

Nombre d'onde $\mathrm{cm}^{-1}$ Quercus $s p \quad$ Abies grandis $L$
Attribution des bandes d'absorption

\begin{tabular}{|c|c|c|}
\hline 3355 & 3355 & Élongation $(\mathrm{O}-\mathrm{H})$ \\
\hline $2933^{e}$ & & Élongations antisymétriques $(\mathrm{CH} 2)$ \\
\hline 2906 & 2900 & Élongations $(\mathrm{C}-\mathrm{H})$ aliphatiques \\
\hline 1734 & 1734 & Élongations $(\mathrm{C}=0)$ \\
\hline 1650 & $1650^{\mathrm{L}}$ & $\begin{array}{c}\text { Élongations massif } 1680(\mathrm{C}=0) \text { cétones conjuguées } \\
1638(\mathrm{H}-\mathrm{O}-\mathrm{H})\end{array}$ \\
\hline 1594 & 1605 & Déformations $(\mathrm{C}=\mathrm{C})$ dans les cycles aromatiques \\
\hline 1504 & 1510 & Déformations $(\mathrm{C}=\mathrm{C})$ dans les cycles aromatiques \\
\hline 1461 & 1455 & Déformations $\left(\mathrm{CH}_{2}\right)$ et $\left(\mathrm{CH}_{3}\right)$ \\
\hline 1423 & 1425 & $\begin{array}{l}\text { Cisaillements }\left(\mathrm{CH}_{2}\right) \text { principalement et }\left(\mathrm{CH}_{3}\right) \\
\text { (lignine) }\end{array}$ \\
\hline 1370 & 1371 & $\begin{array}{l}\text { Déformations }(\mathrm{C}-\mathrm{H}) \text { (cellulose et hémicellulose) } \\
\text { Déformations symétriques }(\mathrm{CH} 3) \text { (lignine) }\end{array}$ \\
\hline $\begin{array}{l}1328 \\
1318 \\
1266\end{array}$ & $1335^{e}$ & $\begin{array}{l}\text { Balancements }\left(\mathrm{CH}_{2}\right) \text { et }(\mathrm{O}-\mathrm{H}) \\
\text { Déformations }\left(\mathrm{CH}_{2}\right) \text { et }(\mathrm{OH}) \\
\text { Élongations }(\mathrm{C}-\mathrm{O}) \text { (lianine et mannanes) }\end{array}$ \\
\hline 1235 & $1230 \mathrm{e}$ & $\begin{array}{l}\text { Déformations }(\mathrm{CH} 2) \text { et }(\mathrm{OH}) \\
\text { Élongations antisymétriques }(\mathrm{C}-\mathrm{O}-\mathrm{C})\end{array}$ \\
\hline 1162 & 1163 & Élongations antisymétriques $(\mathrm{C}-\mathrm{O}-\mathrm{C})$ \\
\hline 1110 & 1110 & Élongations $(\mathrm{C}-\mathrm{O}-$ et $(\mathrm{O}-\mathrm{H})$ \\
\hline 1056 & 1060 & Élongations $(\mathrm{C}-\mathrm{O})$ et $(\mathrm{O}-\mathrm{H})$ \\
\hline 1035 & $\begin{array}{r}1036 \\
863^{\circ}\end{array}$ & $\begin{array}{l}\text { Élongations }(\mathrm{C}-\mathrm{C}) \text { (cycles glucosidiques } \\
\text { Déformations en dehors du plan des }(\mathrm{C}-\mathrm{H}) \\
\text { aromatiques - valence des groupes mannanes }\end{array}$ \\
\hline & 806 & $\begin{array}{l}\text { Déformations des liaisons }(\mathrm{C}-\mathrm{C}) \text { des cycles - } \\
\text { valence des groupes mannanes }\end{array}$ \\
\hline
\end{tabular}

e épaulement; $\llcorner$ large bande.

dans le cas du bois de chêne. Toutefois, quelques différences entre les 2 essences, sont observables :

- alors que dans le cas du bois de chêne, il se produit une baisse générale de l'absorption sur l'ensemble de la région spectrale 1300 à $1450 \mathrm{~cm}^{-1}$, elle a plutôt tendance à augmenter lors de l'irradiation du bois de sapin de Vancouver;

- pour le bois de chêne, l'augmentation à $1735 \mathrm{~cm}^{-1}$ semble résulter de l'apparition de 2 pics différents (1735 et $1720 \mathrm{~cm}^{-1}$ ) (fig 12). Pour le bois de sapin de Vancouver, l'augmentation d'absorption correspond à une seule bande centrée à 1720 $\mathrm{cm}^{-1}$.

\section{Influence des substances extractibles}

La comparaison des comportements des coupes de duramen et d'aubier permet une étude indirecte de l'influence des extraits. Des modifications importantes des quanti- 


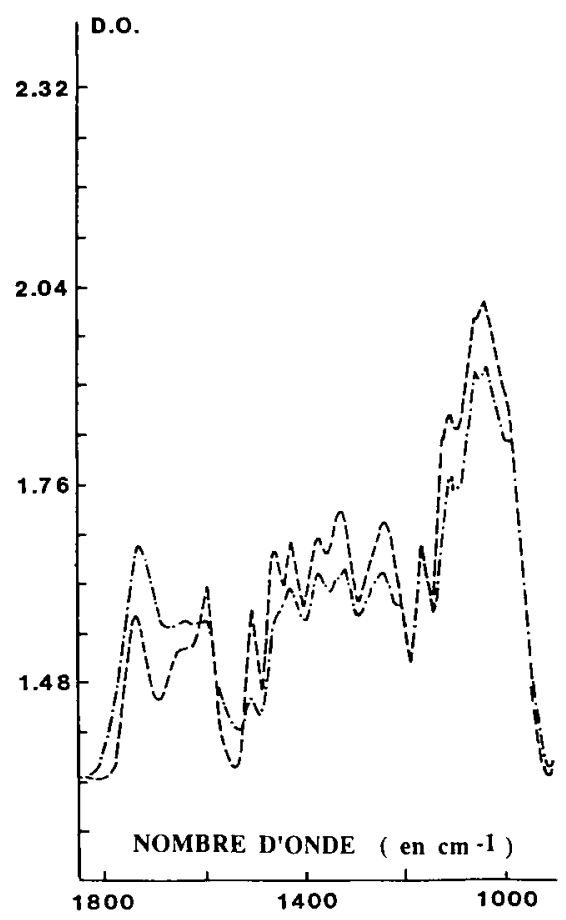

Fig 10. Spectres d'absorption infrarouge de duramen de bois de chêne. --.-- Spectre de départ. .... Spectre après $1700 \mathrm{~h}$ d'irradiation.

tés de polyphénols extractibles se produisent lors du processus de duraminisation. Ainsi, pour le bois de chêne pédonculé, le contenu passe de $0,74 \%$ pour l'aubier à $6,7 \%$ pour le duramen (Scalbert et al, 1986; Scalbert et Monties, 1987). Ces mêmes auteurs ont également montré que ces teneurs ne diffèrent pas de manière significative entre les 2 espèces de bois de chêne (Quercus petraea (Matt) Liebl et Quercus robur L) que nous avons étudiées.

Nous avons déjà noté (fig $2 a$ et b) que l'aubier s'éclaircit plus rapidement et jaunit plus intensément que le duramen. Qualita-
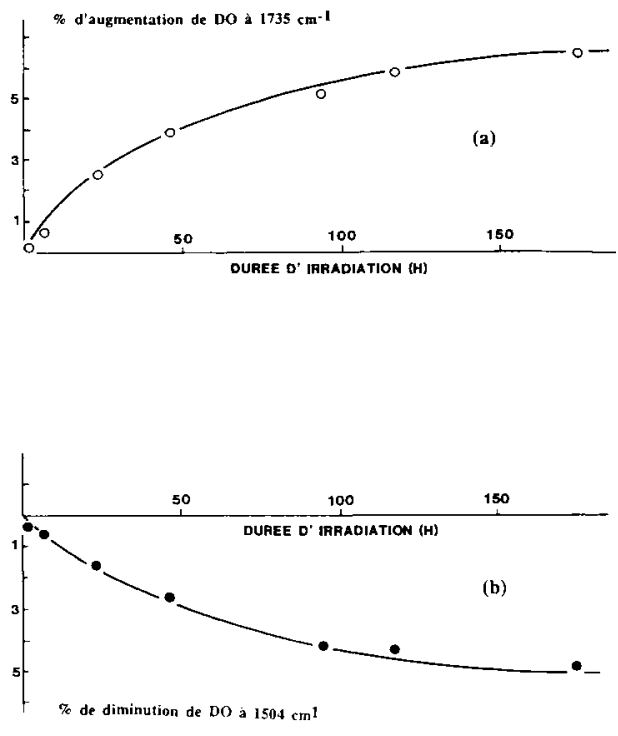

Fig 11. Duramen de bois de chêne : évolution des bandes d'absorption infrarouges situees vers $1735 \mathrm{~cm}^{-1}$ (a) et $1504 \mathrm{~cm}^{-1}$ (b) au cours du temps d'irradiation.

tivement, les évolutions de $L$, a et $B$ vont dans le même sens pour les 2 types d'échantillons. L'aubier et le duramen présentent des évolutions comparables des spectres d'absorption UV-visible au cours de l'irradiation. En début d'exposition, nous avons constaté que l'augmentation de l'absorption sur le domaine visible et particulièrement à $470 \mathrm{~nm}$ s'effectue aussi bien dans le cas de l'aubier que dans le cas du duramen. La diminution de la bande d'absorption vers $340 \mathrm{~nm}$ est plus intense pour l'aubier. De même, en spectroscopie infrarouge, l'irradiation affecte les mêmes bandes d'absorption pour les 2 types d'échantillons. L'étude cinétique de l'évolution pour les 2 types d'échantillons. L'étude cinétique de l'évolution de ces bandes permet de les différencier; ainsi, par exemple, nous avons constaté une plus forte aug- 


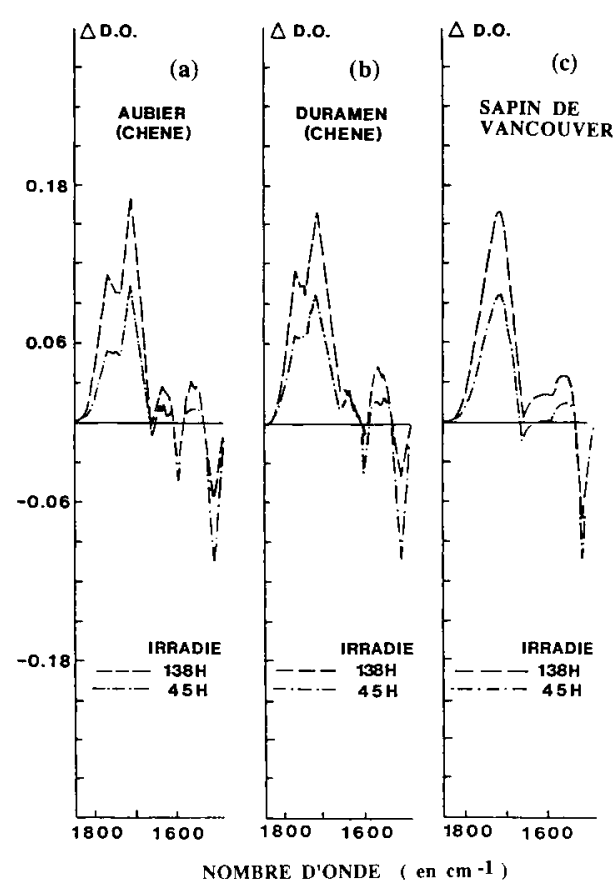

Fig 12. Courbes de différence de spectres infrarouges après/avant irradiation de bois de chêne : aubier (a) et duramen (b), et de bois de sapin de Vancouvert (c).

mentation de la bande à $1735 \mathrm{~cm}^{-1}$ dans le duramen (fig 13).

Cette étude de l'influence des extraits peut également être envisagée en suivant le comportement d'échantillons de bois de chêne extrait à l'eau froide. Après avoir plongé des échantillons massifs d'aubier et de duramen de bois de chêne pendant $4 \mathrm{j}$ dans l'eau à température ambiante, nous les avons soumis à une succession de mises à vide et de remises à l'atmosphère. La figure 14 présente les évolutions de la luminance de ces échantillons au cours de l'irradiation. L'aubier et le duramen ayant subi l'extraction s'éclaircissent plus rapidement que les échantillons témons corres-

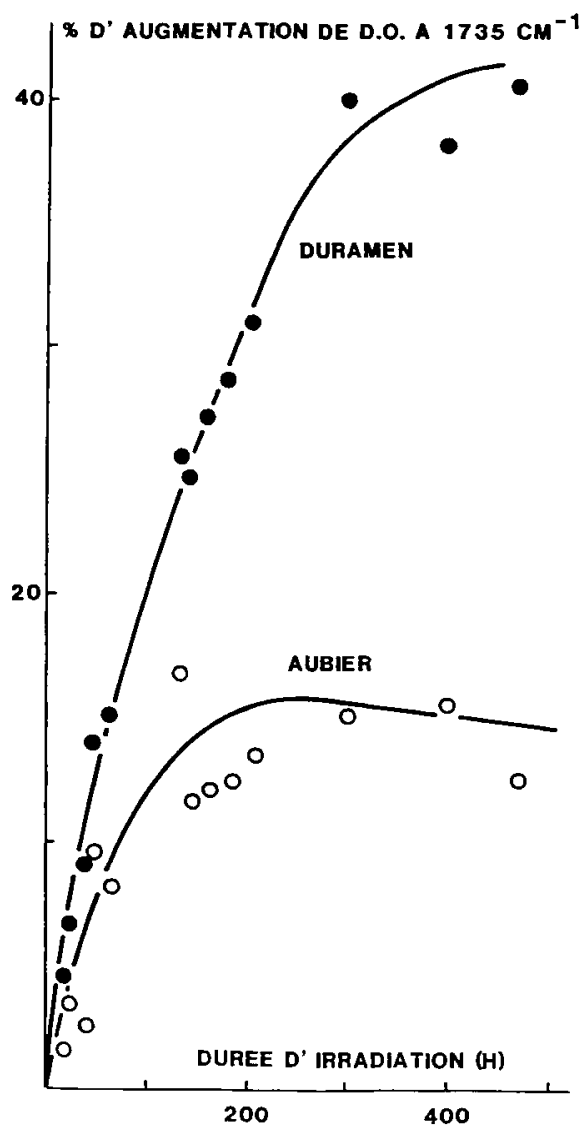

Fig 13. Évolution de la bande d'absorption infrarouge à $1735 \mathrm{~cm}^{-1}$ au fil du temps d'irradiation pour le duramen et l'aubier de bois de chêne.

pondants. Nous pouvons noter en particulier la rapidité avec laquelle l'aubier extrait gagne des points en luminance. L'évolution des coordonnées chromatiques $a$ et $b$ ne permet pas de dégager l'influence de l'extraction, mais elle indique que tous les échantillons extraits ou non jaunissent au cours de l'irradiation.

Ces résultats montrent que le bois de chêne libéré de certaines substances extractibles à l'eau s'éclaircit plus vite que le 


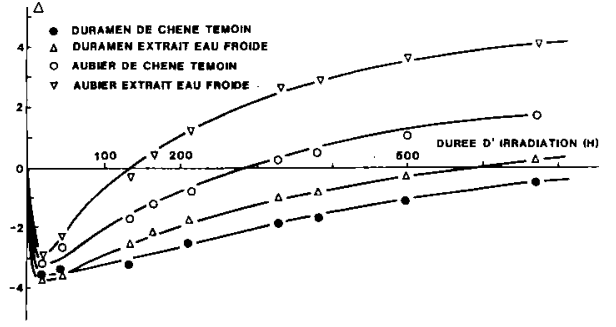

Fig 14. Évolution des caractéristiques de couleur d'échantillons de bois de chêne extraits ou non à l'eau froid (aubier et duramen) en fonction du temps d'irradiation. Duramen de chêne témoin; $\Delta$ duramen extrait eau froid; $O$ aubier de chêne témoin; $\vee$ aubier extrait eau froide.

bois brut. La même observation a été faite sur différentes espèces extraites successivement avec du pétrole, de l'acétone et du méthanol (Morgan et Orsler, 1968). Une étude comparative de l'évolution à l'irradiation de l'aubier et du duramen de mélèze (Larix decidua Mill) (Minemura et Umehara, 1979) a montré d'une part une bande de décoloration plus intense pour les échantillons d'aubier et d'autre part des valeurs de $\Delta \mathrm{E}$ moins élevées lors de l'irradiation d'échantillons de bois de mélèze extrait au méthanol.

Sandermann et Schlumbom (1962) indiquent que les substances extractibles sont impliquées dans les phénomènes de photoéclaircissement des bois. De même, Kalnins (1966), par une analyse chromatographique, mesure, au cours de l'irradiation du bois de sapin de Douglas, des quantités de monoxyde de carbone et de gaz carbonique plus importantes pour les échantillons extraits; il en conclut que les substances extractibles ont un effet protecteur et pourraient fonctionner comme des antioxydants. II est certain que les termes "extraits" ou "substances extractibles" s'appliquent à des produits diffé- rents selon l'espèce de bois considérée et le solvant utilisé pour l'extraction. II faut donc rester prudent quant à la comparaison des résultats obtenus dans toutes ces études.

\section{Influence de la lumière visible}

Des échantillons de duramen et d'aubier de bois de chêne ont été soumis à des irradiations en lumière visible obtenue à l'aide d'un filtre passe-haut coupant à 400 $\mathrm{nm}$. Des échantillons témoins de même nature ont été exposés à la totalité du rayonnement émis par la lampe. La figure 15 montre les évolutions des points de couleur dans le système CIE. Lab des échantillons exposés à un rayonnement filtré ou non. Nous notons que le rayonnement ultraviolet compris entre 300 et 400 $\mathrm{nm}$ influence considérablement le jaunissement des échantillons, ainsi que l'assombrissement en début d'irradiation. En revanche, les vitesses d'éclaircissement après environ $100 \mathrm{~h}$ d'exposition sont du même ordre. II apparaît donc que le photoéclaircissement doit être imputé à la lumière visible. L'analyse des spectres de réflexion ultraviolet-visible de ces échantillons a complété ces observations : la bande de jaunissement nécessite le rayonnement ultraviolet, tandis que la large bande de décoloration centrée à 580 $\mathrm{nm}$ se produit aussi bien avec les échantillons témoins qu'avec ceux irradiés en lumière visible.

En suivant les évolutions des spectres d'absorption infrarouge de coupes d'aubier et de duramen de bois de chêne irradiées par un rayonnement visible (fig 16), nous avons noté que les bandes à $1735 \mathrm{~cm}^{-1}$ et à $1510 \mathrm{~cm}^{-1}$, attribuées respectivement aux groupes carbonyls et aux noyaux aromatiques de la lignine, ne sont pas affectés par ce type de rayonnement: 

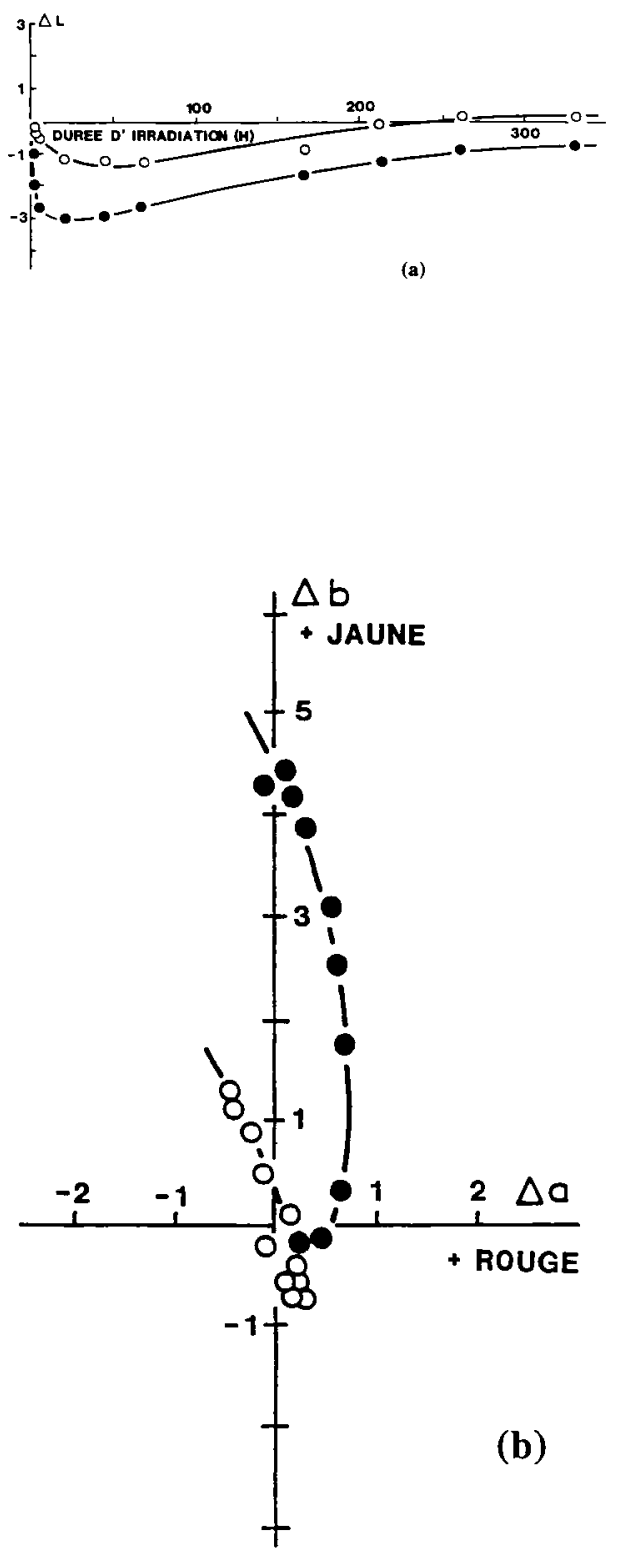

Fig 15. Comparaison des évolutions des caractéristiques de couleur du duramen de bois de chêne soumis à 2 types différents de rayonnement $(\lambda>400 \mathrm{~nm}$ ou $\lambda>290 \mathrm{~nm})$. Duramen de chêne irradié à $\lambda>290 \mathrm{~nm}$; O duramen de chêne irradié à $\lambda>400 \mathrm{~nm}$.
- pour les échantillons témoins, l'absorption à cette longueur d'onde commence par décroître pour ensuite augmenter après $30 \mathrm{~h}$ d'exposition;

- pour les échantillons irradiés uniquement par la lumière visible, nous avons observé une diminution continue et rapide de l'intensité de cette bande. Même après $440 \mathrm{~h}$ d'irradiation, aucune augmentation de l'absorption n'a été détectée. La diminution de l'absorption à $1640 \mathrm{~cm}^{-1}$ observée en début de l'irradiation avec toute la lampe est donc due à l'effet du rayonnement visible, alors que l'apparition des chromophores absorbant dans cette même région spectrale nécessite l'action du proche ultraviolet.

Ces résultats montrent que le rayonnement visible est capable, dans un matériau comme le bois de chêne, de générer une modification de sa couleur, alors que pour le bois d'Abies grandis, ce type de rayonnement est sans action (Dirckx, 1988, op cit). Pour cette essence, seule une diminution de l'absorption à $360 \mathrm{~nm}$ a été observée en spectroscopie d'absorption UVvisible. II faut noter que cette diminution est également observée dans le cas du bois de chêne.

L'étude des modifications de couleur d'autres essences exposées à un rayonnement de type solaire a également montré que la bande de décoloration est induite par le rayonnement visible (Nolan et al, 1945; Sinclair et Vincent, 1964; Umehara et al, 1979; Minemura et Umehara, 1979).

Launer (1968) et Le Nest et al (1982) ainsi que Schmitt (1984) constatent sur divers matériaux lignocellulosiques une diminution de la bande d'absorption centrée sur la longueur d'onde émise par une source monochromatique. Ce phénomène est appelé conformité spectrale. Pour ces auteurs, elle correspond à un véritable blanchiment du matériau si celui-ci est 

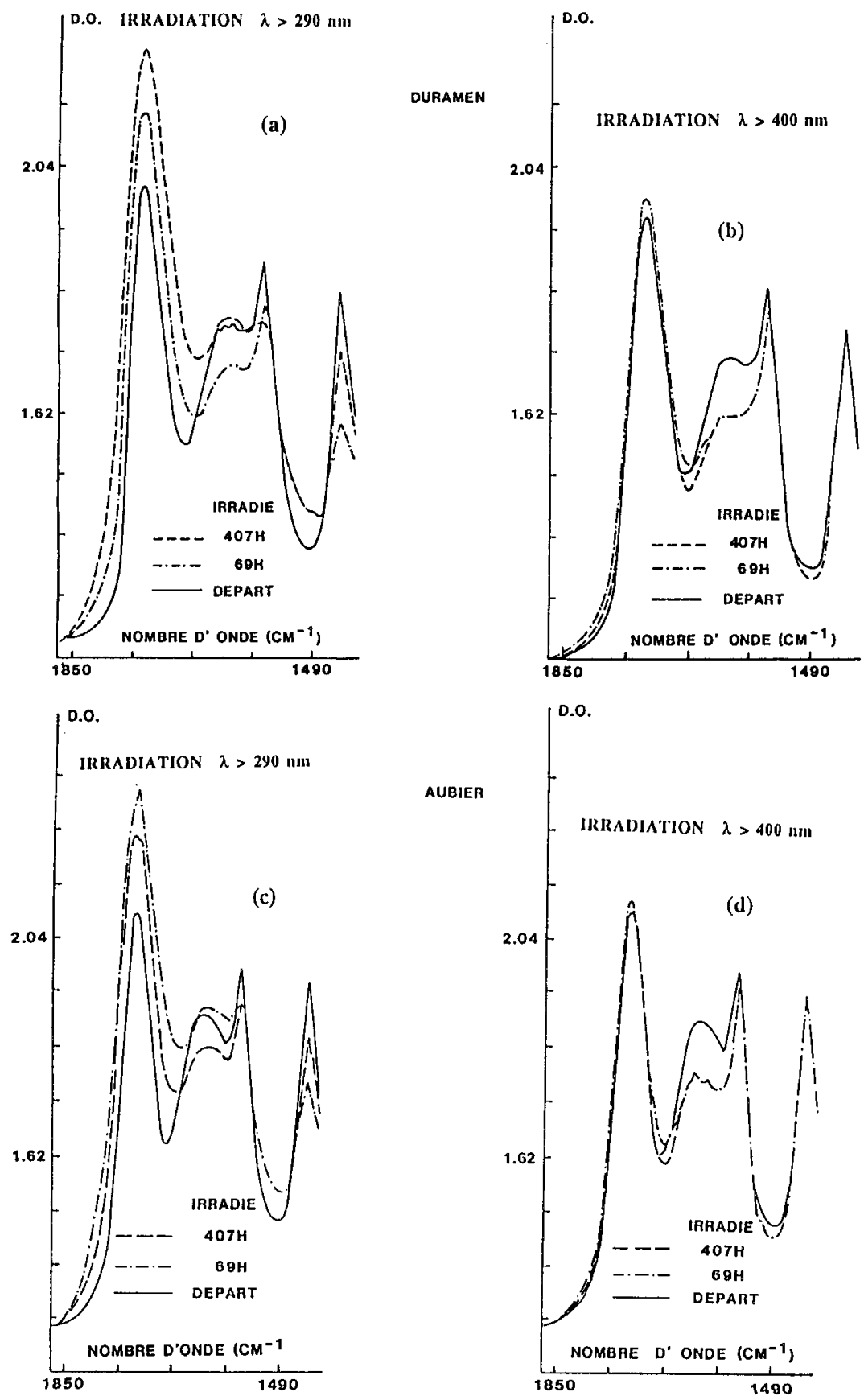

Fig 16. Évolution des spectres infrarouges d'absorption en fonction du type d'irradiation $(\lambda>290 \mathrm{~nm}$ ou $\lambda>400 \mathrm{~nm}$ ) du duramen : (a) et (b) et de l'aubier (c) et (d) de bois de chêne. 
éclairé par un rayonnement polychromatique comme la lumière du jour.

\section{Influence de l'oxygène}

Pour apprécier le rôle de l'oxygène de l'air dans les phénomènes de photodégradation observés sur le bois de chêne, nous avons réalisé des irradiations dans l'air et en atmosphère d'azote. Les courbes de différence des spectres d'absorption ultraviolet-visible des coupes de duramen de bois de chêne (fig 17) montrent que l'absorption à $350 \mathrm{~nm}$ décroît, que l'irradiation soit conduite en présence ou non d'oxygène. De même, en début d'irradiation (fig 17a, b), l'augmentation générale de l'absorption sur l'ensemble du domaine visible (400-700 $\mathrm{nm}$ ) existe dans les 2 cas; nous constatons également que la différence de densité optique à $47 \mathrm{Cm}$ est du même ordre pour les 2 types d'atmosphère (fig 18a). Ce n'est qu'après une quinzaine d'heures d'irradiation qu'apparaît une bande d'absorption centrée à $410 \mathrm{~nm}$ pour l'échantillon irradié en présence d'oxygène (fig 18b). Ce phénomène est bien mis en évidence sur les courbes de différence des spectres (fig 17c, d). Nous notons également que le photoéclaircissement (fig 17c) ne se manifeste que pour l'échantillon irradié en présence d'oxygène.

Le suivi des modifications des spectres infrarouges montre que seule une diminution continue de l'absorption à $1600 \mathrm{~cm}^{-1}$ est observée sur une coupe de bois de chêne irradiée en atmosphère d'azote. Ainsi, l'apparition des groupes carbonyls (augmentation de l'absorption à $1740 \mathrm{~cm}^{-1}$ ) et la dégradation de la structure aromatique des lignines (diminution de l'absorption à $1510 \mathrm{~cm}^{-1}$ ) observées lors d'une irradiation à l'air (fig 11) nécessitent la présence d'oxygène.
Ces résultats montrent que le duramen de bois de chêne irradié en atmosphère d'azote jaunit très peu, l'oxygène de l'air étant nécessaire à la création de produits colorés absorbant vers $410 \mathrm{~nm}$. La même observation a également été faite sur des matériaux lignocellulosiques différents : Abies grandis (Dirckx, 1988; Dirckx et al, 1992); papier journal et Pinus radiata (Leary, 1968a et b); molécules modèles de lignine (Castellan et al, 1985); pâte à papier mécanique (Nolan et al, 1945) et lignine (Lin et Kringstadt, 1971). En revanche, l'oxygène semble sans influence sur l'augmentation générale de l'absorption s'étendant de 400 à $700 \mathrm{~nm}$, et en particulier sur la formation de photoproduits absorbant vers $470 \mathrm{~nm}$. L'augmentation initiale de l'absorption à $430 \mathrm{~nm}$ dans le cas du bois de sapin attribuée à l'apparition de produits radicalaires est également indifférente à la présence ou l'absence d'oxygène (Dirckx et al, 1992). Wengert (1966) fait la même observation en irradiant des échantillons de bouleau : après l'assombrissement de tous les échantillons pendant les premières heures d'exposition, seuls ceux qui sont placés dans l'air et dans l'oxygène pur commencent à s'éclaircir, tandis que ceux exposés en atmosphères inertes (argon, azote et vide) continuent à s'assombrir.

Notons également que la diminution de la densité optique à $350 \mathrm{~nm}$ a lieu dans les 2 atmosphères pour nos échantillons. En rapprochant les observations faites en spectroscopie infrarouge, nous pouvons penser que les produits dont on suit la dégradation vers $350 \mathrm{~nm}$ sont responsables de l'absorption en infrarouge à $1660 \mathrm{~cm}^{-1}$.

\section{DISCUSSION}

La similitude des courbes de différence des spectres d'absorption ultraviolet-visible 

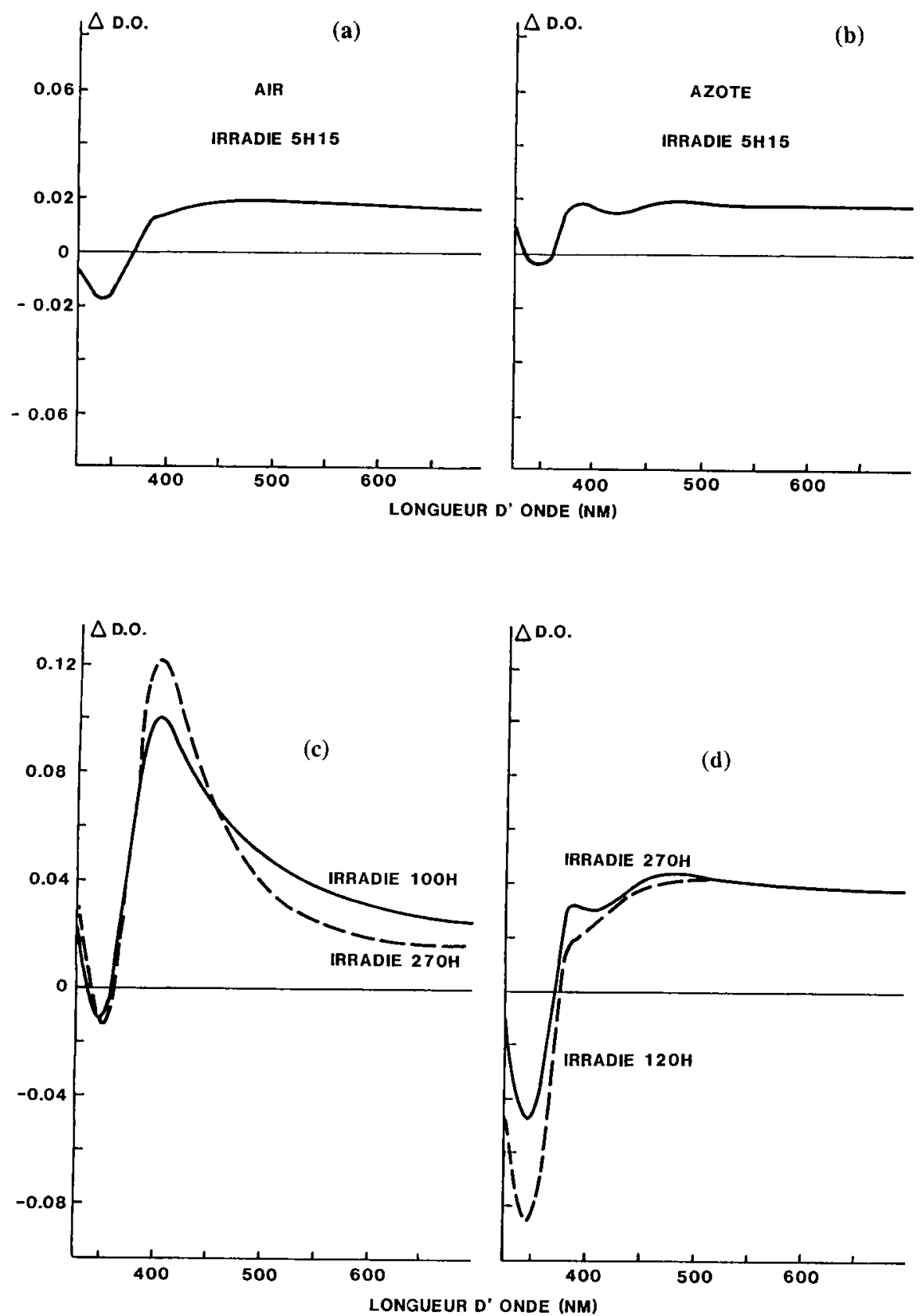

Fig 17. Évolution des courbes de différences de spectres d'absorption ultraviolet-visible de bois de chêne (duramen) irradié en présence d'air : (a) et (c) et dans l'azote : (b) et (d). 

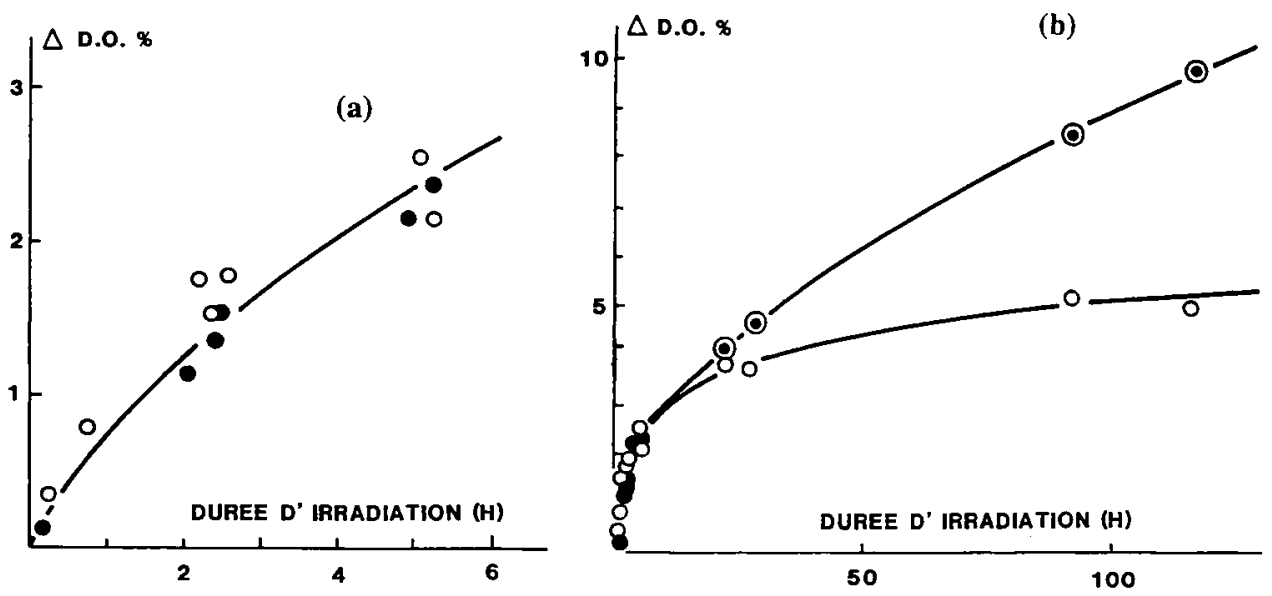

Fig 18. Évolution de la densité optique à $480 \mathrm{~nm}$ au début de l'irradiation sous air et sous azote puis à $410 \mathrm{~nm}$ sous air. Duramen de chêne : DO vers $480 \mathrm{~nm}$ (air); $\Theta$ DO vers $410 \mathrm{~nm}$ (air); $O$ DO vers $480 \mathrm{~nm}$ (azote).

(fig 19) et des spectres d'absorption infrarouge (fig 20) montre que le comportement à la lumière solaire d'une essence feuillue contenant des substances extractibles colorées en forte proportion ne s'éloigne pas trop de celui du bois d'Abies grandis choisi comme modèle du comportement photochimique du bois (Dirckx et al, 1992). Une analyse plus fine permet de dégager 2 différences principales dans l'évolution des 2 essences :

- d'une part, les amplitudes de changement de couleur sont plus faibles dans le cas du bois de chêne (fig 21a);

- d'autre part, il existe, dans les courbes de différence des spectres d'absorption ultraviolet-visible, une large bande de décoloration dans le domaine visible pour le bois de chêne, alors qu'elle est inexistante dans le cas du bois d'Abies grandis. Le phénomène apparaît également dans les évolutions de la luminance (fig 21b) qui, dans le cas du duramen de bois de chêne, augmente après une centaine d'heures d'exposition.
Ce phénomène de jaunissement qui est dû à l'effet des rayonnements ultraviolets a été observé aussi bien sur le bois luimême (Sinclair et Vincent, 1964; Leary, 1967; Dirckx, 1988) que sur les pâtes à papier mécanique (Nolan et al, 1945) ou chimique (Claesson et al, 1967), sur le papier (Leary, 1968a et b; Lenest et al, 1982) et surtout sur des modèles de lignine (Lin et Kringstadt, 1970; Brunow et Eriksson, 1971; Brunow et Sivonen, 1975; Gellerstedt et Pettersson, 1975 et 1977; Vanucci et al, 1987; Castellan et al, 1988). L'ensemble de ces études fait apparaître que, des constituants principaux du bois, ce sont les lignines qui sont responsables du jaunissement du bois à la lumière solaire. Il a été observé que la courbe de sensibilité du bois à la lumière est tout à fait similaire au spectre d'absorption ultraviolet des lignines (Nolan et al, 1945; Dirckx, 1988). Par ailleurs, Scott et Goring (1970) rapportent l'intense décroissance de l'absorption à $280 \mathrm{~nm}$; attribuée aux lignines et observée par microscopie ultraviolette sur les 


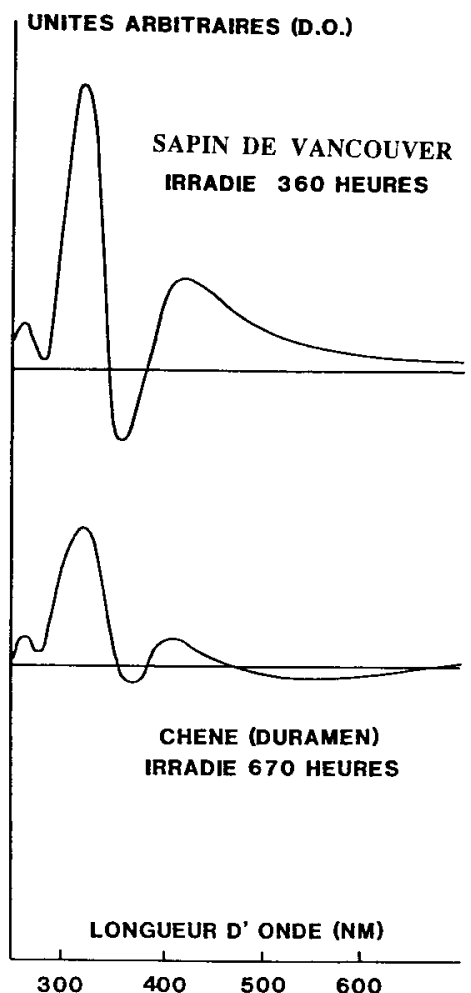

Fig 19. Comparaison des courbes de différences de spectres d'absorption ultravioletvisible après/avant irradiation du bois de chêne et du bois de sapin de Vancouvert.

coupes de bois. Ces études ont également mis en évidence l'importance des groupements $\alpha$-carbonyles conjugués à un cycle aromatique et des groupes cinnamiques dans les phénomènes de photodégradation des lignines. Les réactions induites par l'absorption de la lumière solaire par ces chromophores conduisent à la formation de radicaux phénoxy de type gaïacyl et syringyl, qui sont générés aussi bien en présence qu'en absence d'oxygène (Dirckx, 1988; Dirckx et al, 1992). Les radicaux gaïacoxy absorbent aux environs de
$430 \mathrm{~nm}$ (Neumann et al, 1986a). Par effet bathochrome du substituant, les radicaux de type syringyl absorbent à des longueurs d'onde plus élevées. Ce déplacement peut expliquer l'augmentation progressive de l'absorption à $470 \mathrm{~nm}$ observée en début d'irradiation des coupes de bois de chêne aussi bien dans l'air que dans l'azote. Nous avons par ailleurs vérifié par spectroscopie de résonance paramagnétique électronique la nature radicalaire de la photodégradation du bois de chêne. Un signal de même allure que celui observé lors de l'irradiation du bois d'Abies grandis et de lignine isolée a été détecté. L'étude de la cinétique d'apparition de ces radicaux a montré que l'on obtient un état stationnaire après $1 \mathrm{~h}$ d'irradiation avec un flux lumineux moyen de $20 \mathrm{~mW} / \mathrm{cm}^{2}$ à $360 \mathrm{~nm}$. La longue durée de vie de ces radicaux après arrêt de l'excitation lumineuse est compatible avec une structure phenoxy qui est stabilisée par résonance.

En présence d'oxygène, ces radicaux forment des produits à structure quinonique absorbant vers $400-420 \mathrm{~nm}$, et participant ainsi au jaunissement. L'étude de l'influence de l'oxygène sur le comportement photochimique des lignines a montré que les composés à structure paraquinonique interviennent dans les processus photochimiques de dégradation en sensibilisant la formation d'oxygène singulet. Sous cette forme électroniquement exitée, l'oxygène peut réagir sur les lignines pour reformer des radicaux phénoxy (Neumann et al, 1986b; Neumann et Machado, 1989). En absence d'oxygène, il y a recombinaison des radicaux pour former des produits non colorés (Gellerstedt et Petersson, 1975). Cette désactivation est également observée lors de l'irradiation des molécules modèles précurseurs des lignines en solution dégazée (Castellan et al, 1985). Aucune coloration ne survient lors de l'irradiation. Néanmoins, un jaunissement intervient lorsqu'après exposition, la solution est 


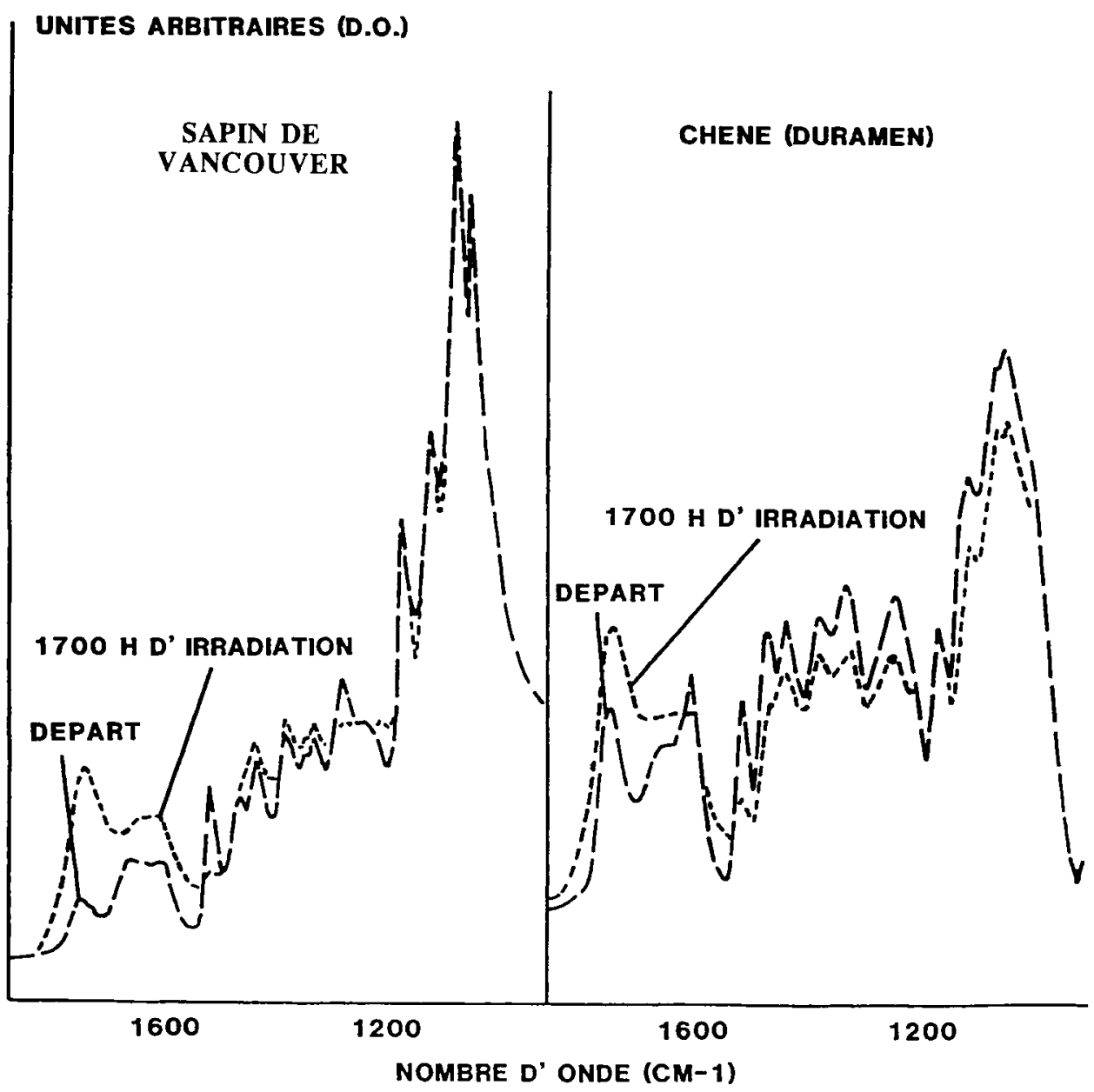

Fig 20. Comparaison des évolutions au cours de l'irradiation des spectres infrarouges du bois de chêne et du bois de sapin de Vancouvert.

stockée à l'obscurité et remise à l'air. Ce résultat peut être interprété en supposant une réaction équilibrée de recombinaison des radicaux de type phénoxy. Après ouverture à l'air, l'équilibre est déplacé vers la formation des espèces radicalaires qui sont consommées pour former les sroduits de type quinonique (fig 22).
Les observations faites sur les coupes de bois de chêne concordent avec ces interprétations : en absence d'oxygène, il n'y a pas d'augmentation importante de l'absorption à $410 \mathrm{~nm}$. En revanche, la croissance à $470 \mathrm{~nm}$ persiste. Nous avons aussi observé que la bande d'absorption à $420 \mathrm{~nm}$ croît lors d'un stockage à l'air et à 

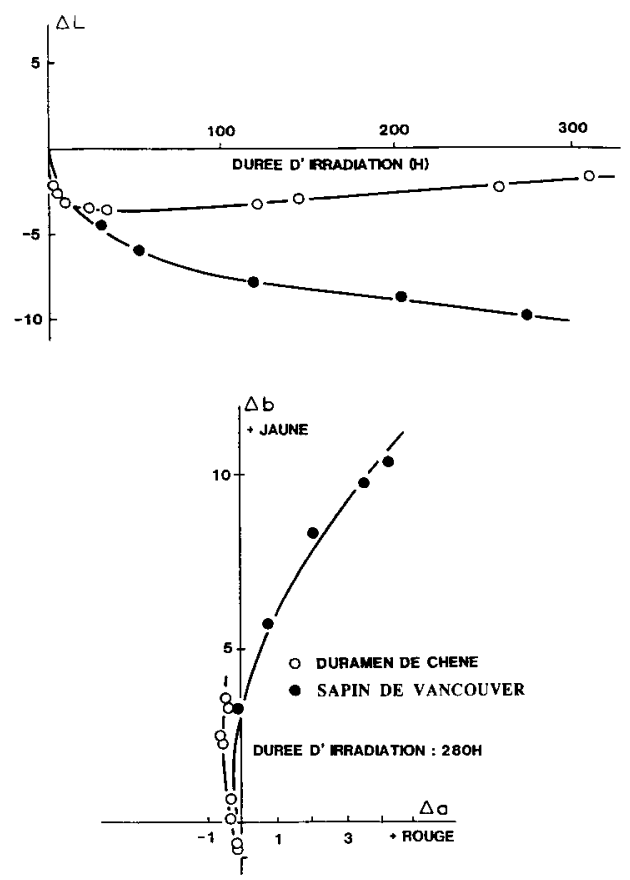

Fig 21. Comparaison de l'évolution au cours du temps d'irradiation des caractéristiques de couleur du duramen de bois de chêne et de bois de sapin de Vancouver. O Duramen de chêne; sapin de Vancouver.

l'obscurité de coupes de bois de chêne préalablement irradiées.

Les groupes phénoliques interviennent également dans le mécanismes de photodégradation du bois. La diminution de l'absorption initiale à $365 \mathrm{~nm}$ reste la même que les hydrogènes mobiles des fonctions phénoliques soient ou non bloqués par acétylation ou méthylation (Leary, 1968). En revanche, ces traitements chimiques inhibent la bande de jaunissement centrée aux environs de $410 \mathrm{~nm}$. Ces observations montrent que les groupements phénoliques ne jouent aucun rôle dans l'absorption initiale du rayonnement ultraviolet,

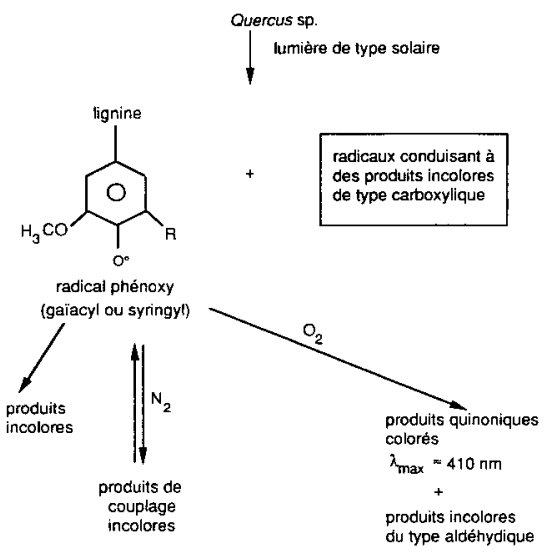

Fig 22. Désactivation des radicaux phénoxy en présence et en absence d'oxygène.

mais participent sans aucun doute ultérieurement au processus de jaunissement.

Le rôle des phénols dans la photodégradation des lignines n'est pas encore clairement élucidé (Castellan et al, 1990; Heitner et Schmidt, 1991). L'étude de la photolyse en solution des molécules modèles des lignines a montré que la présence de groupes phénoliques libres n'est pas nécessaire pour induire une forte coloration (Vanucci et al, 1988). Kringstadt et Lin (1970) notent que le blocage des hydrogènes des groupes phénoliques ne permet pas une stabilisation totale de la couleur du papier de pâtes à haut rendement. En effet, les groupes carbonyles excités par la lumière sont susceptibles d'arracher un atome d'hydrogène mobile aux polysaccharides provoquant ainsi un jaunissement à l'air.

Pour tenter d'expliquer le phénomène de photoéclaircissement observé sur le bois de chêne et inexistant sur le bois d'Abies grandis, il apparaît important de re- 
chercher ce qui diffère entre les compositions chimiques de ces 2 essences :

- les substances extractibles : de l'ordre de $7 \%$ en poids (total des extraits éthanolbenzène, eau) pour les bois d'Abies balsamea et d'Abies sachalinensis alors qu'elles atteignent 11 à $13 \%$ pour le bois de Quercus robur (Lal et al, 1977; Fengel et Wegener, 1984); pour ces 2 genres, ces substances sont des terpènes, des graisses et cires, des composés phénoliques comme les lignanes, les stilbènes, des flavonoïdes; pour le bois de chêne, elles sont en plus constituées par des extraits colorés dont les plus importants sont les tanins essentiellement hydrolysables ou ellagiques (Scalbert et al, 1987);

- la structure des lignines : la structure des lignines de feuillus contient un nombre plus important d'unités syringyles (Nimz, 1974) alors qu'il y a beaucoup plus de gaïacyls pour les résineux (Adler et Marton, 1959; Adler, 1977). Cette différence est d'ailleurs la seule détectée en spectroscopie infrarouge entre les bois de chêne et de sapin (fig 9).

De plus, la quantité de lignine est plus importante pour les différentes espèces d'Abies (entre 27 et $30 \%$ ) que pour le genre Quercus en général (22 à $25 \%$ ) (Fengel et Wegener, 1984; Dirckx et al, 1992).

À partir de ce bilan des différences de composition chimique entre les essences feuillues et résineuses, nous sommes tentés d'attribuer la bande de décoloration observée pour le bois de chêne aux substances extractibles colorées, et en particulier aux tanins. La détermination de la structure chimique de ces composés (Mayer et al, 1967a,b; 1969; 1971a,b) montre que ces tanins sont des polyphénols présentant une forte délocalisation électronique et absorbant dans la zone spectrale du visible. En étudiant la photochimie en solution des substances extrac- tibles du bois de chêne, nous avons observé que ces composés sont peu stables à la lumière; une irradiation à l'air par un rayonnement de type solaire provoque une décoloration de la solution. Ce résultat montre que les substances extractibles coIorées jouent un rôle déterminant dans les processus d'éclaircissement. Toutefois, si seuls ces composés étaient responsables de ce jaunissement, comment expliquer que l'aubier de bois de chêne s'éclaircisse plus vite que le duramen ou qu'un échantillon extrait à l'eau froide présente une bande de décoloration plus intense qu'un échantillon non traité ? Comme le jaunissement, le photoéclaircissement doit être imputé au moins en partie à la lignine ellemême. Ce phénomène est d'ailleurs observé lors de l'irradiation des lignines de bois d'Abies grandis obtenues par une extraction au dioxanne (Dirckx, 1988) et de bois de Fagus sylvatica L (Schmitt, 19841). La participation des lignines dans l'absorption du bois dans le visible, donc à sa couleur, a été établie pour diverses essences. Une relation linéaire entre le contenu en lignine de Klason et le coefficient d'absorption à $457 \mathrm{~nm}$ est observée sur le bois de pin (Pinus spp) (Wilcox, 1975) et il a pu être montré que cette absorption dans le visible devait être attribuée aux chromophores de type coniféraldéhyde présents dans les lignines (Norsstrom, 1969; Pew et Connors, 1971; Hon et Glasser, 1979). Ainsi, au cours de l'étude de blanchiment du duramen et de l'aubier du bois de sapin par du peroxyde d'hydrogène, une forte décroissance de l'absorption à $340 \mathrm{~nm}$; corrélée à la fois avec une diminution à $280 \mathrm{~nm}$ (caractéristique des lignines) et avec une baisse de la densité optique à 400 et à $457 \mathrm{~nm}$ est constatée (Douek et Goring, 1976). Le chromophore absorbant à 340 $\mathrm{nm}$ pourrait, selon ces auteurs, être attribué au groupe coniféraldehyde. II apparaît donc établi que la disparition de ce groupement appartenant aux lignines suivie à 340 
nm entraîne une diminution de l'absorption dans le visible.

II faut noter que tous ces travaux concernent les lignines de résineux riches en unités du type coniféraldéhyde. Les lignines de feuillus contiennent quant à elles autant de groupements coniféraldéhydes que sinapaldéhyde. Un substituant méthoxy supplémentaire sur le cycle aromatique (par exemple en passant du coniféraldehyde ou sinapaldéhyde) déplace les bandes d'absorption ultraviolet-visible vers les grandes longueurs d'onde. Ainsi, la queue de la bande centrée vers $350 \mathrm{~nm}$ empiète plus dans le domaine visible (fig 23) (Pew et Connors, 1971). Nos résultats montrent d'ailleurs que cette bande de décoloration survient même lors d'une irradiation en lumière visible $(\lambda>400 \mathrm{~nm})$. Or le rayonnement visible possède une énergie inférieure à $70 \mathrm{kcal} / \mathrm{mol}$ incapable de rompre directement des liaisons chimiques dans le bois (Hon et Ifju, 1978). Il est alors possible que la lumière visible excite certains chromophores du bois, qui se désactivent en induisant des réactions secondaires avec une diminution de la conjugaison des électrons. II en résulterait ainsi un éclaircissement du matériau.

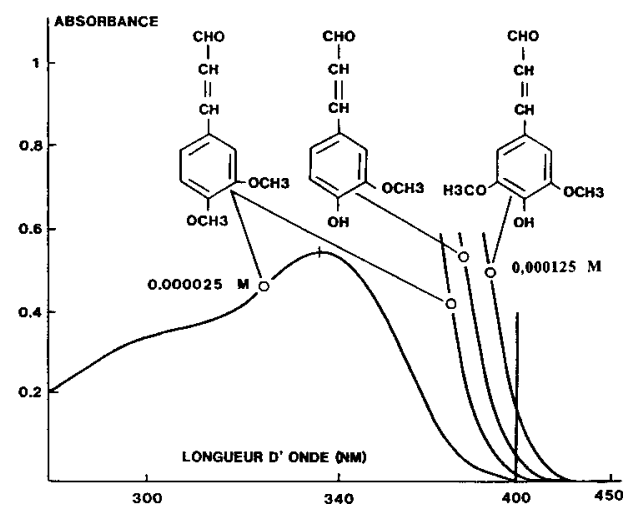

Fig 23. Spectres d'absorption ultraviolet-visible du conyféraldéhyde et du synapaldéhyde.
Nos résultats ont également montré que ces réactions nécessitent la présence d'oxygène, le phénomène de décoloration n'étant pas détecté dans le cas d'une irradiation en atmosphère d'azote.

Ce phénomène a également été observé sur des lignines isolées et sur différentes essences de bois lors d'une irradiation par un rayonnement proche ultraviolet (300 nm à $400 \mathrm{~nm}$ ) (Wengert, 1968; Schmitt, 1984). Ce domaine spectral recouvre bien celui de l'absorption des chromophores de type cinnamique (coniférylique ou sinapylique).

Cette bande de décoloration existe également dans le cas du bois d'Abies grandis mais n'est pas détectable, compte tenu de l'importance considérable du jaunissement. En effet, un traitement par du polyéthylèneglycol qui inhibe la bande de jaunissement située à $410 \mathrm{~nm}$, permet de révéler le phénomène d'éclaircissement. L'intensité de la bande de jaunissement du bois d'Abies grandis est accentuée par rapport à celle observée sur les lignines extraites de cette essence, sur cette dernière, la bande de décoloration est directement détectée (Dirckx, 1988). On peut penser que, dans le cas du bois d'Abies grandis, des extraits non colorés mais absorbant dans le proche ultraviolet participent au jaunissement. En effet, les stilbènes (Morgan et Orsler, 1968) et les triterpènes cycliques insaturés (Bergman, 1969) sont impliqués dans la photodégradation de certains bois exotiques ou de la pâte à papier.

\section{CONCLUSION}

L'objectif de cette étude était, sur la base d'un travail à caractère fondamental réalisé sur le bois d'Abies grandis L (Dirckx, 1988; Dirckx et al, 1992) d'apporter une contribution à une meilleure compréhension à l'échelle moléculaire des mécanismes de 
la photodégradation du bois, en s'intéressant au comportement photochimique du bois de chêne, pour lequel la définition des critères de qualité passe par des caractéristiques de couleur.

Nous avons montré qu'une essence riche en substances extractibles colorées comme le bois de chêne présente un comportement photochimique peu différent de celui du bois d'Abies grandis. Les différences les plus marquées se situent dans les intensités des modifications spectrales, plus importantes pour le bois de sapin et dans la présence d'un éclaircissement (djminution de l'absorption de 500 à $600 \mathrm{~nm}$ ) observé uniquement dans le cas du bois de chêne et attribué, comme le jaunissement, aux lignines.

Les essais réalisés ont également permis de mettre en évidence les comportements photochimiques différents de l'aubier et du duramen de bois de chêne.

Il se dégage de nos résultats que la stabilisation de la couleur d'un échantillon de bois de chêne soumis à une irradiation de type solaire ne peut être totale qu'en envisageant l'action combinée de substances absorbeur-UV pour freiner le jaunissement provoqué par le rayonnement ultraviolet et de substances antioxydantes pour limiter la décoloration, qui n'intervient qu'en présence d'oxygène.

\section{RÉFÉRENCES}

Adler E, Marton J (1959) Zur Kenntnis der Carbonylgruppen im Lignin. I. Acta Chem Scand 13, 75-96

Adler E (1977) Lignin chemistry: past, present and future. Wood Sci Technol 11, 169-218

Bergman J (1965) Yellowing of the pulp extractives. Sven Papperstidn 68, 339-347

Bird RC, Hulstrom RL, Kliman AW, Eldering HG (1982) Solar spectral measurements in the terrestrial environment. Appl Optics 21(8), $1430-1431$
Bolker HI, Sommerville NG (1963) Infrared spectroscopy of tignins. Part II: Lignins in unbleached pulps. Pulp and paper magazine of $\mathrm{Ca}$ nada $64,187.194$

Browne FL, Simonson HC (1957) The penetration of light into wood. For Prod J October 57 . 308-314

Brunow G, Eriksson B (1971) $\alpha$ carbonyl groups as sensitizers in the photo dehydrogenation of phenolic structures in lignin. Acta Chem Scand 25(7), 2779-2781

Brunow G, Sivonen M (1975) The yellowing of lignin. Part II: the participation of oxygen in the photo-deshydrogenation of lignin model compounds. Papper och Trä, special number $4 a$, 215-220

Castellan A, Vanucci C, Desvergne JP, Bouas Laurent $H$, Hauteville $M$, Chadenson $M$ (1985) Étude photochimique de dimères modèles de la lignine de type $\alpha-0-4$ et $\beta-0-4-0$ méthylés. CR Acad Sci Paris II, 301, 21-23

Castellan A, Girard P, Vanucci C (1988) A photoyellowing simulation of bleached high-yield wood pulp by lignin models included in solid carbohydrate matrix. $J$ Wood Chem Technol 8(1), 79-90

Castellan A, Colombo N, Nourmamode A, Zhu $J H$, Lachenal $D$, Davidson RS, Dunn L (1990) Discoloration of $\alpha$-carbonyl-free lignin model compounds under UV light exposure. J Wood Chem Technol 10(4), 461-477

Chow SZ (1971) Infrared spectra characteristics and surface inactivation of wood at high temperatures. Wood Sci Technol 5, 27-39

Claesson J, Olson E, Wennerblom A (1967) The yellowing and bleaching by light of lignin-rich papers and the re-yellowing in darkness. Sven Pappertsidn 71 (8), 335-340

Decarreau JF (1988) Le système CIE Lab. Bull Sci Couleur 1, 13-15

Dirckx O (1988) Étude du comportement photochimique de l'Abies grandis sous irradiation solaire. Thèse de $3^{\circ}$ cycle en sciences du bois, Université de Nancy I

Dirckx O, Mazet JF, Merlin A, Deglise X (1990) Approche de la stabilisation de la couleur du bois exposé à la lumière. Actes du $3^{e}$ colloque sciences et industries du bois, Bordeaux 14-15 mai 1990 , tome I, 181-190

Dirckx O, Triboulot-Trouy MC, Merlin A, Deglise $X$ (1992) Étude des modifications de la cou- 
leur de l'Abies grandis exposé à la lumière solaire. Ann Sci For 5, 425-448

Dordet Y (1990) La colorimétrie. Principe et applications. Eyrolles, Paris

Douek M, Goring DAI (1976) Microscopial studies of the peroxide bleaching of Douglas Fir wood. Wood Sci Technol 10, 29-38

Fengel D, Wegener G (1984) Wood: chemistry, ultrastructure, reactions. De Gruyter, Berlin

Elot JL (1988) La couleur du chêne de tranchage français. Rapport de stage ENITEFINRA, station de recherches sur la qualité du bois, centre de Champenoux

Gaillard JM (1984) Photodégradation de systèmes bois-finitions (résines alkydes). Thèse de $3^{\text {e }}$ cycle en sciences du bois, University de Nancy 1

Gellerstedt G, Petterson EL (1975) Light induced oxydation of lignin. Part I: The behaviour of structural units containing a ring conjugated double bond. Acta Chem Scand B29(10), 1005-1010

Gellerstedt G, Petterson EL (1977) Light induced oxydation of lignin. Part II: The oxidative degradation of aromatic rings. Sven Papperstidn 80(1), 15-21

Heitner C, Schmidt JA (1991) Light-induced yellowing of wood containing papers. A review of fifty years of research. Proc 6th int Symposium of Wood and Pulp Chem, Melbourne (Australie) 1, 131-149

Hon DNS (1983) Weathering reactions and protection of wood surfaces. $J$ Applied Polymer Sci (Applied Polymer Symposium) 37, 845864

Hon DNS, Chang ST (1984) Surface degradation of wood by ultraviolet light. $J$ Polymer Sci 22, 2227-2241

Hon DNS, Ifju G (1978) Measuring penetration of light into wood by detection of photoinduced free radicals. Wood Sci 11(2), 118-127

Hon DNS, Glasser W (1979) On possible chromophoric structures in wood and pulps: a survey of the present state of knowledge. Polym Plast Technol Eng 12(2), 159-179

Janin $G$ (1986) La couleur du bois, un facteur clé de qualité. Mesures 1, 61-65

Kalnins AM (1966) Surface characteristics of wood as they affect durability of finishes. Part II. Photochemical degradation of wood. US For Sen Res Paper FPL 57, 23-60
Kringstadt KP, Lin SY (1970) Mechanism in the yellowing of high-yields pulps by light. Structure and reactivity of free radical intermediates in the photodegradation of lignin. Tappi 53 (12), 2296-2301

Lablache-Combier A (1985) Photochimie et polymères. Actual Chim, 39-60

Lal NK, Butnaru R, Simionescu C (1977) Investigation of the field of chemical and morphological structure of certain tropical-wood species. II: The study of their chemical composition. Cellul Chem Technol 11, 59-66

Launer $F$ (1968) Spectral conformity on widespread effect of light. Nature 218 (13), 161 163

Leary GJ (1967) The yellowing of wood by light. Tappi 50 (1), 17-19

Leary GJ (1968a) The yellowing of wood by light. Part II. Tappi 51(6), 257-260

Leary GJ (1968b) Photochemical production of quinoïd structures in wood. Nature 217, 672673

Le Nest JF, Silvy J, Gandini A (1982) Effets des radiations visibles et proches ultraviolettes sur les matériaux lignocellulosiques. Actes du $1^{\text {er }}$ colloque sciences et industries du bois, Grenoble, 367-384

Lin SY, Kringstadt KP (1970) Photosensitive groups in lignin model compounds. Tappi 53 (4), 658-663

Lin SY, Kringstadt KP (1971) Some reactions in the photoinduced discoloration of lignin. Norsk Skogindustri 25(9), 252-256

Marchessault RH (1962) Application of infrared spectroscopy to cellulose and wood polysaccharides. Pure Applied Chem 5, 107-129

Mayer W, Gabler W, Riester A, Korger H (1967a) Über die Gerbstoffe aus Holz der Edelkastanie und der Eiche, II: Die Isolierung von Castalagin, Vescalagin, Castalin und Vescalin. Liebigs Ann Chem 707, 177-181

Mayer W, Einwiller A, Jochims JC (1967b) Über die Gerbstoffe aus Holz der Edelkastanie und der Eiche, III: Die Struktur des Castalins. Liebigs Ann Chem 707, 182-189

Mayer W, Seitz H, Jochims JC (1969) Über die Gerbstoffe aus Holz der Edelkastanie und der Eiche, IV: Die Struktur des Castalins Liebigs Ann Chem 721, 186-193

Mayer W, Kuhlmann F, Schilling G (1971a) Über die Gerbstoffe aus Holz der Edelkastanie und 
der Eiche, V: Die Struktur des Vascalins. Liebigs Ann Chem 747, 51-59

Mayer W, Seitz H, Jochims JC, Schauerte K, Schilling G (1971b) Über die Gerbstoffe aus Holz der Edelkastanie und der Eiche, VI: Die Struktur des Vescalagins. Liebigs Ann Chem $751,60-68$

Mazet JF (1988) Couleur et qualité des placages de chêne et étude de leur comportement photochimique. Thèse de Doctorat en sciences du bois, Université de Nancy I

Mc Ginnes EA (1975) Influence of incandescent and fluorescent light on the color of unfinished heartwood of black walnut and eastern red cedar. Wood Sci 7(4), 270-279

Michell AJ, Ingle HD, Steward CM (1969) Infrared spectra of some woods of taxonomic interest. Wood Sci Technol 3, 93-99

Minemura N, Umehara K (1979) Color improvement of wood (Report 1): photoinduced discoloration and its control. I Hokkaïdo For Prod Res Instit 54(3), 92-145

Morgan JWW, Orsler RJ (1968) The chemistry of colour changes in wood. I. The significance of stilbene. Holzforschung 22(1), 11-16

Musha $Y$, Goring DAl (1975) Distribution of syringyl and gaïacyl moieties in hardwoods as indicated by ultraviolet microscopy. Wood Sci Technol 9, 45-58

Nagaty A, El Sayed QH, Ibrahim ST, Mansour OY (1982) Chemical and spectral studies on hemicellulose isolated from bagasse. Holzforschung 36, 29-35

Neumann MG, De Groot RA, Machado AE (1986a) Flash photolysis of lignin. Part I: Deaerated solutions of dioxane-lignin. Polym Photochem 7, 401-403

Neumann MG, De Groot RA, Machado AE (1986b) Flash photolysis of lignin. Part II: Oxidative photodegradation of dioxane-lignin. Polym Photochem 7, 461-468

Neumann MG, Machado AE (1989) The role of oxygen in the photodegradation of lignin in solution. J Photochem Photobiol B Biol 3, 473-481

Nimz H (1974) Beech lignin: proposed of a constitutional scheme. Angew Chem Int Ed Eng 13(5), 313-321

Nolan P, Van Den Akker JA, Wink KA (1945) The fading of groundwood by light. Pap Trade J $121(10), 101.105$
Norsstrom $H$ (1969) Light absorbing properties of pulp and pulp components. Sven Papperstidn 72(2), 25-38

Pecina H (1982) Zur Aussagefähigkeit von Infrarot-Spektrogrammen über chemische Strukturveränderungen des Holzes mit dem Beispiel thermischer Behandlung. Holztechnologie 23(2), 78-84

Pew JC, Connors JW (1971) Color of coniferous lignin. Tappi 54(2), 245-251

Sandermann W, Schlumbom F (1962) Über die Wirkung gefielterten ultravioletten Lichten auf Holz, Erste Mitteilung: Photometrische und chromatographische Untersuchungen an Holzmehlen. Holz als Roh und Werkstoff, Heft 7, 285-291

Scalbert A, Monties B (1987) Comparison of the lignin and phenolic contents in the heartwood and spawood of oaks (Quercus robur and petraea). Communication orale du 10/12/197, INRA, centre de Grignon, groupe de travail interlaboratoire

Scalbert A, Monties B, Dupouey JL, Becker M (1986) Polyphénols extractibles du bois de chêne : variabilitè interspécifique, interindividuelle et effet de la duramenisation. Communication présentée aux Journées internationales d'étude du groupe polyphénols, Montpellier, 9-11 juillet 1986

Schmitt $P$ (1984) Étude des effets du rayonnement de la lumière du jour sur la cellulose et les matériaux ligneux. Thèse de $3^{e}$ cycle, INP Grenoble

Scott JAN, Goring DAI (1970) Photolysis of wood microsections in the ultraviolet microscope. Wood Sci Technol 4, 237-239

Sinclar RM, Vincent TA (1964) Yellowing of Radiata pine timber. $N Z J$ Sci 7, 196-206

Umehara K, Minemura N, Suganuma T (1979) Photoinduced discoloration of Northern red oak. J Hokkaido For Prod Res Instit 327,1516

Vanucci C, Girard P, Bouas-Laurent H, Castellan A (1987) Aspects moléculaires de la photodégradation de la lignine. Actes du $2^{\mathrm{e}}$ colloque sciences et industries du bois, Nancy, tome II, 221-228

Vanucci $C$, Forniet de Violet $P$, Bouas-Laurent H, Castellan A (1988) Photodegradation of lignin: a photophysical and photochemical study of a non-phenolic $\alpha$-carbonyl $\beta-0-4$ li- 
gnin model dimer 3-4 dimethoxy-a-(2' methoxyphenoxy) autophenone. J Photochem Photobiol A Chem 41, 251-265

Wengert EM (1966) Effect of atmospheric gases on color changes in wood exposed to ultraviolet light. J Paint Technol 38(493), 71-76

Wilcox MD (1975) Measuring the brightness, light absorption coefficient and light scattering coefficient of wood. Sven Paperstidn 78(1), 22-26 